University of Louisville

ThinkIR: The University of Louisville's Institutional Repository

Electronic Theses and Dissertations

$5-2021$

\title{
Estimating cumulative incidence rate on interval censored data in an illness-death model.
}

Chen Qian

University of Louisville

Follow this and additional works at: https://ir.library.louisville.edu/etd

Part of the Biostatistics Commons

\section{Recommended Citation}

Qian, Chen, "Estimating cumulative incidence rate on interval censored data in an illness-death model." (2021). Electronic Theses and Dissertations. Paper 3636.

https://doi.org/10.18297/etd/3636

This Doctoral Dissertation is brought to you for free and open access by ThinkIR: The University of Louisville's Institutional Repository. It has been accepted for inclusion in Electronic Theses and Dissertations by an authorized administrator of ThinkIR: The University of Louisville's Institutional Repository. This title appears here courtesy of the author, who has retained all other copyrights. For more information, please contact thinkir@louisville.edu. 


\title{
ESTIMATING CUMULATIVE INCIDENCE RATE ON INTERVAL CENSORED DATA IN AN ILLNESS-DEATH MODEL
}

\author{
By \\ Chen Qian \\ B.A., Rhodes College, 2015 \\ M.S., University of Louisville, 2016 \\ A Dissertation \\ Submitted to the Faculty of the \\ School of Public Health and Information Sciences \\ of the University of Louisville \\ in Partial Fulfillment of the Requirements \\ for the Degree of \\ Doctor of Philosophy \\ in Biostatistics \\ Department of Bioinformatics and Biostatistics \\ University of Louisville \\ Louisville, Kentucky
}

May 2021 



\title{
ESTIMATING CUMULATIVE INCIDENCE RATE ON INTERVAL CENSORED DATA IN AN ILLNESS-DEATH MODEL
}

\author{
By \\ Chen Qian \\ B.A., Rhodes College, 2015 \\ M.S., University of Louisville, 2016 \\ A Dissertation Approved on \\ March 31, 2021
}

by the following Dissertation Committee:

Shesh N. Rai, Ph.D., Dissertation Director

Matthew Cave, M.D.

Riten Mitra, Ph.D.

Subhadip Pal, Ph.D.

Elizabeth Riley, M.D.

Qi Zheng, Ph.D. 


\title{
DEDICATION
}

This dissertation is dedicated to my parents

\author{
Mr. Jianping Qian
}

$\&$

Mrs. Youmei Wang

for their endless love, support, and encouragement throughout my life. 


\section{ACKNOWLEDGMENTS}

I would like to thank my advisor Dr. Shesh N. Rai for his unwavering support over the past four and half years. I sincerely appreciate his advice and guidance during my academic journey. I would like to thank my committee members Drs. Matthew Cave, Riten Mitra, Subhadip Pal, Elizabeth Riley and Qi Zheng for their time and valuable input.

With gratitude to Dr. Jianmin Pan and Dr. Deo Kumar Srivastava for their tremendous support throughout my research and special thanks to Dr. Craig McClain for his generous financial support. I appreciate all the faculty members and staff in the Department of Bioinformatics and Biostatistics for their support.

I also want to thank the Nanik family (Jerry, Lois and Larry from Verndale, MN) and the Brezina family (Connie, Tony, and their lovely kids from New Prague, MN) for their enormous love and support in my education journey.

I want to conclude my thanks to Mrs. Yunfei Li and Dr. Cheng Liu for their immense support, along with my parents, relatives and all my friends who have enhanced my life. 


\section{ABSTRACT \\ ESTIMATING CUMULATIVE INCIDENCE RATE ON INTERVAL CENSORED DATA IN AN ILLNESS-DEATH MODEL \\ Chen Qian}

March 31, 2021

Phase IV clinical trials are designed to monitor long-term side effects caused over time by the medical treatment. For instance, in advanced primary cancer treatment, childhood cancer survivors are often at risk of developing undesired events, such as cardiotoxicity, during their adulthood. Such problems could be due to their cancer or the treatment they received for their cancer such as radiation or intensive chemotherapy.

Cardiotoxicity can be diagnosed with electrophysiology with measurements of fraction shortening, afterload, etc. Often the primary focus of a study could be on estimating the cumulative incidence (CI) of a particular outcome of interest such as cardiotoxicity. However, it is not possible to evaluate patients on a continuous basis and often this information is collected through cross-sectional surveys by following patients longitudinally. This leads to interval censored data since the exact time of the onset of toxicity is not known.

This dissertation consists of three projects related to the estimation of cumulative incidence rates on interval censored data in an illness-death model. In the first project, missing observation problem in the current status data is discussed. An imputation method is proposed to handle such issue. The second project introduces 
a new method for estimating transition intensity probabilities using semi-parametric with EM (expectation-maximization) approach in a special case two-state model. A logit relationship for the treatment intensities in the two groups is proposed. In the third project, the cumulative incidence rates are evaluated using the maximum likelihood estimation approach in a complete three-state illness-death model in which death is incorporated as competing risk. 


\section{TABLE OF CONTENTS}

PAGE

DEDICATION

ACKNOWLEDGMENTS $\quad$ iv

ABSTRACT V V

LIST OF TABLES Ix

LIST OF FIGURES $\quad$ x

CHAPTER 1: INTRODUCTION 1

1.1 Imputing Partial Status and Estimating Incidence Rate in an Illnessdeath Model with Application to a Phase IV Cancer Trial . . . . . . 1

1.2 Estimating Transition Intensity Rate on Interval Censored Data using Semi-parametric with EM Algorithm Approach . . . . . . . . . . . 2

1.3 Estimating Cumulative Incidence Rate on Interval Censored Data in an Illness-Death Model . . . . . . . . . . . . . . . . . . . . . . . 3

1.4 Figures . . . . . . . . . . . . . . . . . . 4

CHAPTER 2: IMPUTING PARTIAL STATUS AND ESTIMATING INCIDENCE RATE IN AN ILLNESS-DEATH MODEL WITH APPLICATION TO A PHASE IV CANCER TRIAL 4

2.1 Introduction . . . . . . . . . . . . . . . . . . 5

2.1.1 Background ................... 5

2.1.2 Motivation Example . . . . . . . . . . . . . . 7

2.2 Method . . . . . . . . . . . . . . . . . 10

2.2.1 Cardio-Measures Abnormality Model . . . . . . . . . . . . . . 10

2.2.2 Imputation Model . . . . . . . . . . . . . . . . . . . . . . 10

2.3 Result . . . . . . . . . . . . . . . . . . . . . 12

2.3.1 Application: Cancer Survivor Study . . . . . . . . . . . . . 12

2.3.2 Simulation Study . . . . . . . . . . . . . . . . . 14

2.4 Discussion . . . . . . . . . . . . . . . . . . . . . . 18

2.5 Tables and Figures . . . . . . . . . . . . . . . . . . 20

CHAPTER 3: ESTIMATING TRANSITION INTENSITY RATE ON INTERVAL CENSORED DATA USING SEMI-PARAMETRIC WITH EM ALGO-

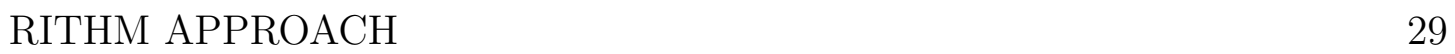

3.1 Introduction . . . . . . . . . . . . . . . . . . 30

3.1.1 Background ....................... 30 
3.1 .2 Methodology Review . . . . . . . . . . . . . . . . . . . . 32

3.1 .3 Motivating Example . . . . . . . . . . . . . . . . . . 33

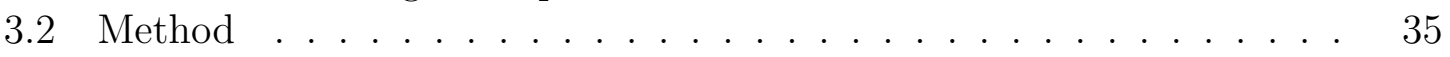

3.2 .1 Overall Flow of the Analysis . . . . . . . . . . . . . . . 35

3.2 .2 Detailed Steps of the Analysis . . . . . . . . . . . . . . 36

3.3 Simulation Studies . . . . . . . . . . . . . . . . . . . . . . 43

3.3.1 Simulation Steps . . . . . . . . . . . . . . . . . . 43

3.3.2 Simulation Results . . . . . . . . . . . . . . . . . . 44

3.4 Discussion . . . . . . . . . . . . . . . . . . . . . . . . . . . . . 45

3.5 Tables and Figures $\ldots \ldots \ldots \ldots \ldots \ldots$

CHAPTER 4: ESTIMATING CUMULATIVE INCIDENCE RATE ON INTERVAL CENSORED DATA IN AN ILLNESS-DEATH MODEL 51

4.1 Introduction . . . . . . . . . . . . . . . . . . . 51

4.1 .1 Background . . . . . . . . . . . . . . . . . 51

4.1 .2 Methodology Review . . . . . . . . . . . . . . . . . . 52

4.2 Method .............................. 53

4.3 Data Application . . . . . . . . . . . . . . . . . 54

4.3 .1 Data Information . . . . . . . . . . . . . . . . . . . 54

$4.3 .2 \quad$ Application Result . . . . . . . . . . . . . . . . 55

4.4 Discussion . . . . . . . . . . . . . . . . . 56

4.5 Tables and Figures $\ldots \ldots \ldots \ldots \ldots \ldots$

$\begin{array}{ll}\text { FUTURE WORK } & 61\end{array}$

REFERENCES 62

$\begin{array}{ll}\text { APPENDIX } & 71\end{array}$

$\begin{array}{ll}\text { CURRICULUM VITA } & 74\end{array}$ 


\section{LIST OF TABLES}

TABLE PAGE

2.1 Statistical Summary of the Patient Population in the Cardiac Study. 23

2.2 Likelihood Contributions Corresponding to Different Observation Types. 24

2.3 Coefficients and p-values in Regression Model. . . . . . . . . . . . . 25

2.4 AF p-values for Group Effect using Likelihood Ratio Test. . . . . . . 26

2.5 p-values for Group Effect using Likelihood Ratio Test $(n=180) \ldots . . . \quad 27$

2.6 p-values for Group Effect using Likelihood Ratio Test $(n=240) \ldots$. . . 28

2.7 p-values for Group Effect using Likelihood Ratio Test $(n=300) \ldots . . . \quad 29$

3.1 Comparison of simulated mean $\lambda$ and $\beta$ values with the true value. . 49

3.2 Confidence intervals, interquartile range, and standard deviations for $\lambda$ and $\beta$ with different sample size. . . . . . . . . . . . . . . 50

4.1 Transition intensity rates for AR and NR groups. . . . . . . . . . . 59

4.2 Fixed-term cumulative incidence rates for AR and NR groups. . . . . 60 


\section{LIST OF FIGURES}

FIGURE

PAGE

1.1 Three-state illness-death model. . . . . . . . . . . . . . . . . .

2.1 Scatter plot of AF and FS within AR and NR group of patients. Complete data (labeled c) displays strong correlation between AF and FS. Missing values of AF are in almost the entire range of FS values (labeled $\mathrm{m}$ ).

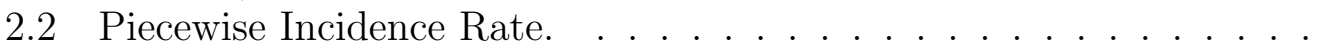

2.3 Cumulative Incidence Comparison for AF Based on Original Data and Imputed Data (Using the mean of intensity estimates) Corresponding to $\mathrm{m}=5,20$ and 100. Note: The lines for $\mathrm{m}=5$ and 20 are almost

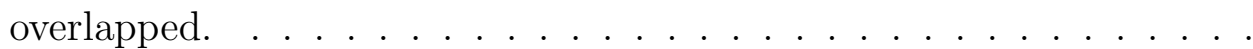

3.1 Corresponding $\lambda$ values with different sample size assumptions (AR group). . . . . . . . . . . . . . . . . .

3.2 Corresponding $\lambda$ values with different sample size assumptions (NR group). . . . . . . . . . . . . . . . . . 48

4.1 Fixed-term cumulative incidence rates for AR and NR groups $\left(\lambda_{1}\right) . \quad$. 58 


\section{CHAPTER 1 INTRODUCTION}

This dissertation will focus on estimating fixed-term cumulative incidence rates in an illness-death model. Figure 1.1 provides a visualized overview of the model. The target population in our research is childhood cancer survivors treated with anthracycline chemotherapy and/or thoracic irradiation. The methodology can be extended to other types of interval censored data as well. Cancer survivors are alive and have not developed cardiac abnormality at the initial state (state 1). They can either be diagnosed cardiac abnormality at the follow up (state 2) or reach the terminal state directly with or without cardiac failure (state 3 ). For patients at state 2, they can either remain at the current state when the data is observed or reach the terminal state. Note that for patients at state 2 , the actual onset times $u$ of these events prior to the current status are unknown. Cardiac abnormality can occur any time between state 1 and state 2 . In this dissertation, our primary interest is on estimating

the transition intensity rate $\lambda_{1}(u)$. This will provide valuable information and help evaluate the side effects of the treatment in the long run.

\subsection{Imputing Partial Status and Estimating Incidence Rate in an Illness-death Model with Application to a Phase IV Cancer Trial}

The first project mainly focuses on the missing observation problem in the current status data. One such study was designed by Hudson et al. (2007) to study the effect of anthracyclines exposure, received as part of treatment for childhood cancer, 
to cardiotoxicity. Rai et al. (2013) utilized a parametric approach for assessing the effect of anthracycline on the cumulative incidence of cardiotoxicity but excluded the patients with missing information. In this project, we resort to impute the missing current status using the regression method in an illness-death/failure model. A comprehensive simulation study suggests that the results obtained using the imputation approach are significantly more efficient than those obtained without imputation. We

further apply the proposed approach to the data reported in Rai et al. (2013) and compare the results reported there to our approach that utilizes imputation.

\subsection{Estimating Transition Intensity Rate on Interval Censored Data using Semi-parametric with EM Algorithm Approach}

Rai et al. (2013) computed the transition intensity rate using parametric approach through the maximum likelihood estimation in a special case illness/death model. The anthracycline effect was calculated using the difference of likelihood ratio test. However, the parametric approach may not be suitable if the underlying parametric assumptions do not hold. In the second project, we propose a semi-parametric model, with logit relationship for the treatment intensities in the two groups, to compare the treatment effects within the context of illness-death model. The estimation of the parameters is done using EM algorithm with profile likelihood. Results from simulation studies suggest that the semi-parametric model with EM algorithm approach is practicable and comparable to the parametric model. 


\subsection{Estimating Cumulative Incidence Rate on Interval Censored Data in an Illness-Death Model}

In the third project, a new dataset is being used, which is an updated version of the first data that was discussed in the first project. Since the follow up time is much longer, a handful of death cases have occurred. Follow Rai et al. (2013)'s methodology on two-state models, we extend the application to a complete three-state illness/death model. We resort to incorporate death as competing risk and calculate the cumulative incidence rates using the maximum likelihood estimation approach. 


\subsection{Figures}

Figure 1.1: Three-state illness-death model.

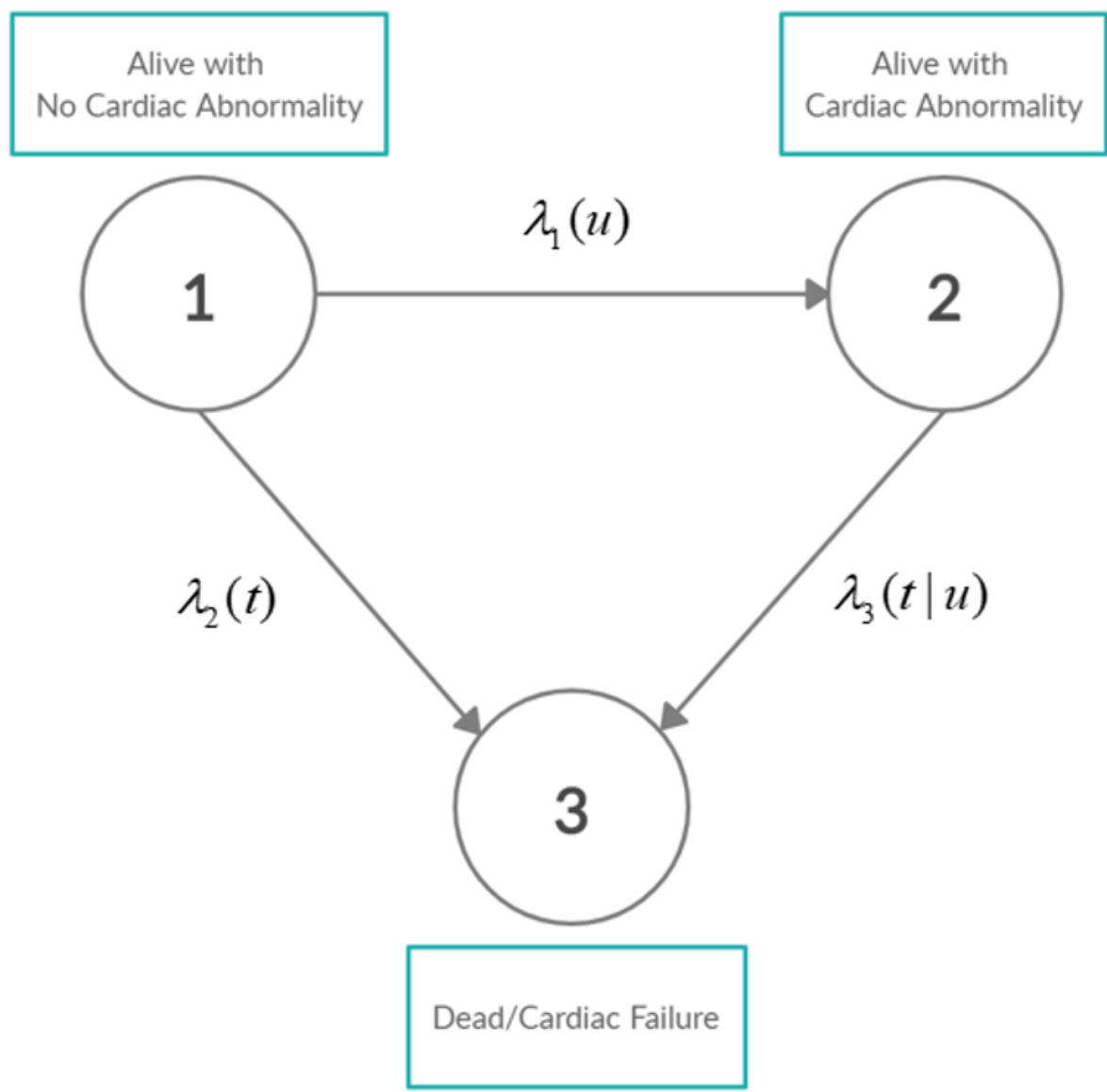

$\lambda_{1}(u)$ is the transition intensity from state 1 to state $2 . \lambda_{2}(t)$ is the transition intensity from state 1 to state 3. $\lambda_{3}(t \mid u)$ is the transition intensity from state 2 to state 3 . 
CHAPTER 2

IMPUTING PARTIAL STATUS AND ESTIMATING INCIDENCE

\section{RATE IN AN ILLNESS-DEATH MODEL WITH APPLICATION \\ TO A PHASE IV CANCER TRIAL}

\subsection{Introduction}

\subsubsection{Background}

As a result of more modern therapies and better supportive care, the 5-year survival rate for childhood cancer has improved significantly and currently exceeds $80 \%$ (Armstrong et al., 2016; Howlader et al., 2015; Phillips et al., 2015; Fidler et al., 2016). In 2011 there were 390,000 survivors of childhood cancer living in the US and it is expected that by 2020 there will be more than 500,000 childhood cancer survivors (Robison and Hudson, 2014). This improvement in survival rate comes at a price as these survivors are at an elevated risk of experiencing long-term morbidity and early mortality as a result of their cancers and its treatment. The purpose of Phase IV clinical trials is to monitor long-term sequela and develop interventions to mitigate their effect in long-term. A chemotherapy agent Anthracycline has served as the backbone for many pediatric malignancies because of its therapeutic effects, but it is also well known to be cardiotoxic (Krischer et al., 1997; Sorensen et al., 2003). One such study was undertaken by Hudson et al. (2007) to evaluate the effect of anthracycline exposure on cardiotoxicity using non-invasive modalities.

Cardiotoxicity is the occurrence of heart electrophysiology dysfunction or/and 
muscle damage. The heart is too weak to pump and circulate blood. There are many measures of electrophysiology dysfunction or/and muscle damage, including shortening fraction, afterload, ejection fraction, etc. (Hudson et al., 2007; Sorensen et al., 2003; Pein et al., 2004). Just like many other types of disease, it is not possible to evaluate patients very often to estimate the onset time of cardiotoxicity partially due to economic concerns. The incidence rates are therefore difficult to estimate. Usually, patients are followed longitudinally in the clinics, but not all follow a routine pattern. Hence, it is convenient to design cross-sectional surveys for estimating the effect of long-term side effect of treatments and its predictors. We only know the current status of the patient with onset prior to current status, but not the actual onset times of these events. These types of incomplete data are referred to as interval censored data since the actual onset time of the events are unknown (Rai et al., 2013; Krischer et al., 1997; Sorensen et al., 2003; Pein et al., 2004; Sun, 2006) and our interest is in the estimation of the onset rate or the cumulative incidence rate.

Nonparametric procedures for analyzing interval censored failure time data have been extensively studied and discussed in the literature (Sun, 2006; Ayer et al., 1955; Van Eeden, 1956; Peto, 1973; Turnbull, 1976). Another issue in the crosssection survey study is that results need to be generalized to the specific population. There can be competing toxic effects from the same drug. In this paper, we have the same interest, as Rai et al. (2013), in estimating the cumulative incidence rates using a parametric approach, but focus on improving the accuracy by imputing the missing observations using multivariable regression method.

In practice, most investigators exclude observations with missing values and incomplete cases. Despite its simplicity as using only complete cases, it may lose the important information in the incomplete cases and ignore the possible systematic differences between the complete and incomplete cases. Hence, the resulting inference may not be applicable to the population of all cases, especially with a smaller 
number of complete cases. It is well known that imputation is a widely used method for handling missing data. Little and Rubin (2002) and Buuren (2012) provided an excellent overview of the methods for conducting analyses with missing data. Further information on multiple imputations can refer to Rubin $(1987,1993)$, and Rubin, Stern, and Vehovar (1995) which discussed imputation of missing discrete data. King et al. (2001) reviewed many of the practical costs and benefits of multiple imputations. For routine imputation of missing data, Schafer (1997) presented a method based on multivariate normal distribution. Liu (1995) used the $t$ distribution, and Van Buuren, Boshuizen, and Knook (1999) used interlocking regressions. Furthermore, Troxel, Ma, and Heitjan (2004) presented a method to study the sensitivity of inferences to missing-data assumptions.

This chapter is organized as follows. In the following subsection, we provide the details of the motivating example to introduce the problem. In the Methods section, we give a brief description of the procedure introduced in Rai et al. (2013) and construct corresponding likelihood functions. The data from the motivation example is analyzed by imputing the missing values and compared with the results obtained without imputation in the Results section. An extensive simulation experiment to study the performance of the imputation approach are summarized as well. The Discussion section is devoted to miscellaneous remarks.

\subsubsection{Motivation Example}

A study was undertaken by Hudson et al. (2007) to evaluate the effect of anthracycline on cardiotoxicity using 12-lead ECG and echocardiography, non-invasive technique. In the study, the cancer survivors were recruited from St. Jude Children's Research Hospital After Completion of Therapy Clinic. The survivors were classified into two groups; the first group consisted of survivors that received cardiotoxic therapy (anthracycline and/or thoracic radiation) and the other group did not receive cardiotoxic 
therapy (no anthracycline nor thoracic radiation). The details of the study can be found in Hudson et al. (2007). The study was approved by the institutional review boards at St Jude Children's Research Hospital and Stanford University (Stanford, CA). All study participants or their parents provided informed consent. Survivors with cardiotoxic therapy were designated as At-Risk (AR) and those without cardiotoxic therapy as Not At-Risk (NR).

One-time clinical assessment was made by the primary oncologists to identify the survivors with signs of heart failure using New York Heart Association classification. Along with the clinical evaluation, non-invasive testing based on 12-lead ECG and echocardiography were conducted within 24 hours of the clinical assessment.

In this project, the outcome variables we used are afterload (AF) and fractional shortening (FS). A short summary about the cohort is summarized in Table 2.1. Further description is available in Hudson et al. (2007). Detailed explanation of a similar motivation example can be found in Rai et al. (2013). On closer examination of the data, it was seen that there were missing data in outcome measures $\mathrm{AF}$ and FS. A total of 40 survivors had missing AF values and 6 of those were also missing FS values. To keep the discussion simple and straightforward these 6 observations were deleted from our analysis and our attention was paid to imputing 34 missing AF observations. Although, the imputation approach discussed could easily be applied to data missing with several variables in a recursive manner. The scatter plot of AF and FS displayed in Figure 2.1 does not show any clear missing pattern; the missing values of $\mathrm{AF}$ are in the entire range of values of FS. Also note that the missing proportion of $\mathrm{AF}$ values in the $\mathrm{NR}(7 / 54=13 \%)$ and $\mathrm{AR}(27 / 218=12 \%)$ were almost identical.

In this study, echocardiography was performed as a research measure and not in response to clinical symptoms. Individuals with previously established cardiac disease were excluded from participation. As formally assessed by New York Heart 
Association classification, none of the study participants reported clinical symptoms of cardiac dysfunction at enrollment. The imaging quality in echocardiography is dependent on obtaining a clear acoustic window from which ventricular volumes are estimated based on geometric assumptions. Operator experience and variations in thoracic structures can contribute to difficulties in obtaining technically satisfactory data in a given study. These factors randomly contributed to missing data among study participants. AF is not a standardly used assessment in clinical practice, thus, despite training of ultrasonographers for this study, this factor may have contributed to a higher prevalence of missing AF measurements compared to FS. In other words, AF is either under detected or over detected, but operators might miss it, and therefore, causing missing values. Thus, we feel that there is no selection bias related to those with and without abnormal FS and AF identified as part of the study. The missing values of AF are displayed in Figure 2.1, also do not show any pattern.

A crude approach to estimating the incidence rates and obtaining confidence intervals is to use the Kaplan-Meier estimator with the assumption of the evaluation time as the onset time. Then, the incidence rate of each type of toxicity is estimated. Some of these toxicity measures could be missing. In this project, we impute the missing measurements using a regression method first and then use a parametric approach to estimate incidence rates of specific toxicity. For the cardiotoxicity data, the 34 missing AF values were imputed using a multivariable regression with FS and other covariates, such as age, diagnosis, risk status, BMI as predictors. The estimates of cumulative incidence rates were derived based on the data after imputation and then compared with those derived in Rai, et al. (2013) without imputation. 


\subsection{Method}

\subsubsection{Cardio-Measures Abnormality Model}

The descriptive statistics of all participants can be found in Table 2.1. Detailed cardiomeasures abnormality model can be found in Rai et al. (2013). The general theory for a cross-sectional data with indicators of cardiac abnormality and death/cardiac failure, and time since the treatment to the survey or the death/cardiac failure, as depicted in Figure 1.1, can be found in Rai et al. (2013) as well. We also assume that cardiac abnormality is irreversible and is the precursor for cardiac failure.

We are interested in estimating $\Lambda_{1}(t)=\int_{0}^{t} \lambda_{1}(u) d u$, the cumulative incidence function (CIF), but focus on the comparison of CIFs between AR and NR groups based on the original data and imputation data. $\lambda_{1}(u)$ is the transition intensity for patients progress from state 1 to state 2 .

Table 2.2 identifies the various types of observations which occur in this illnessdeath/failure model and the corresponding contribution to the likelihood, denoted as $L_{1}(t)$ to $L_{4}(t)$, which are functionals of intensities and pseudo-survival functions. The approach of the likelihood functions can be found in Appendix 1. Rai et al. (2013) derive the explicit form of $L_{1}(t)$ to $L_{4}(t)$ for both constant and piecewise exponential models and the likelihood functions. $X(t)$ represents the state that the patient is current occupying. $Q(t)$ represents the probability that the time to the first event exceeds $t$.

\subsubsection{Imputation Model}

Let the cardiac measure, such as AF, be denoted by $Y$. Assume that $Y=\left(Y_{1}^{T}, Y_{2}^{T}\right)$ be a $n \times 1$ response vector with $Y_{1}\left(n_{1} \times 1\right)$ observed and $Y_{2}\left(n_{2} \times 1\right)$ missed, and $X=\left(X_{1}, X_{2}\right)$ be corresponding $n \times p$ matrix comprised of covariates including other cardiac measures (other response variables). 
There are several methods for imputation which can be broadly classified as single imputation or multiple imputation (MI). In the MI approach several copies of the complete data set are created and then the appropriate statistical method is applied to each data set and the results from these analyses are then combined to provide the final results. Usually, MI approaches are preferred over single imputation as they incorporate variability due to imputation (Burren, 2002; Molenberghs and Fitzmoaurice, 2009; Kenward and Carpenter, 2009). There are many MI approaches discussed in literature, but two most commonly used approaches based on joint multivariate modeling or fully conditional specification perform quite well in the regression setting, cited in Huque et al. (2018). It may be noted that PROC MI can perform imputations for data that have monotone or arbitrary missing patterns. PROC MI with FCS option, a standard feature in SAS version 9.4 (SAS Institute, Cary, NC), utilizes the conditional distribution and can incorporate both continuous and categorical variables appropriately, see Liu and De (2015). In our setting, we had missing values only in $\mathrm{AF}$ and we wanted to take advantage of the relationship between $\mathrm{AF}$ and other covariates of interest that included categorical variables. Therefore, we preferred to perform the imputations using PROC MI in SAS with FCS option. The method can be briefly described as follows:

A multivariable regression model $y=x \beta+\epsilon$ is fitted based on the complete data $Y_{1}$ and $X_{1}$, and the least squared estimator $\hat{\beta}$ of $\beta(p \times 1)$ and associated variance-covariance matrix is obtained. Then, missing values in $Y_{2}$ are imputed using the posterior predictive distributions, see PROC MI in SAS (SAS Institute, Cary, $\mathrm{NC})$ for details. It is natural to use the imputation data $\left(Y_{1}, \hat{Y}_{2}\right)^{T}$ instead of only $Y_{1}$

and is anticipated that the imputed information in $\hat{Y}_{2}$ will improve the related results in statistical analysis.

To each complete data set, the likelihood ratio test was applied to compare the two risk groups (AR and NR). In the regression framework, one could use PROC 
MIANALYZE in SAS to combine the results from multiple imputations to conduct inference that incorporates inherent variability introduced due to imputations (Burren, 2002; Molenberghs and Fitzmoaurice, 2009; Kenward and Carpenter, 2009). However, in our setting, p-values associated with each imputation are obtained based on the likelihood ratio test. Then, the overall conclusion can be drawn from some types of summary measure for all the p-values such as mean or median. We prefer to report the results based on median as that would be much more robust than mean.

\subsection{Result}

\subsubsection{Application: Cancer Survivor Study}

In this section, we obtained the imputed data for the cardiotoxicity example and applied the theory for the exponential model (detail can be found in the third project) to evaluate the effect of anthracyclines on cardiotoxicity. Furthermore, the results obtained using the imputation approach were then compared with those obtained without imputation, reported in Rai et al. (2013), under the assumption of no deaths/cardiac failures. The simplest model is the Parameter-1, which is a one param-

eter Exponential model. Normally cardiotoxicity would not occur within five years of cancer treatment. Based on this consideration, we also fit two piecewise Exponential models. Parameter-2, based on two incidence rates in which the first one represents up-to year five and the second one represents year 5 and above, and Parameter-3, based on three incidence rates: up-to year 5 , between year 5 and year 10, year 10 and above (see Figure 2.2 for a general idea).

In the cardiotoxicity example, there are 278 subjects, Leukemia $(n=77)$, Sarcoma $(\mathrm{n}=74)$, Lymphoma $(\mathrm{n}=56)$ and Embryonal $(\mathrm{n}=71)$, and 34 measurements were missing for AF and 6 were missing in both measurements AF and FS, but no covariate 
information was missing. We exclude the 6 with both missing. Hence, we have 272 subjects and employ the multivariable regression method to estimate the 34 missing measurements in AF based on the values of FS and corresponding covariates, like age at diagnosis, race, gender, BMI, QTC, diagnosis group and risk group (AR/NR) (Henry, Gardin and Ware, 1980; Colan et al., 1992). Based on 4 diagnosis groups, we define three dummy variables as follows:

$$
\text { Diag } 1=\left\{\begin{array}{l}
1 \text { Leukemia } \\
0 \text { Otherwise }
\end{array}, \text { Diag } 2=\left\{\begin{array}{l}
1 \text { Sarcoma } \\
0 \text { Otherwise }
\end{array}, \text { Diag } 3= \begin{cases}1 & \text { Lymphoma } \\
0 & \text { Otherwise }\end{cases}\right.\right.
$$

Before conducting the regression analysis, the Shapiro-Wilk test of normality was applied to original AF and FS measurements and a few commonly used transformations for making the underlying distributions of AF and FS more normal. $\log (\mathrm{AF})$ was normally distributed $(\mathrm{p}=0.701)$, but original AF $(\mathrm{p}<0.001)$ was not. On the other hand, $\log (\mathrm{FS})$, Logit(FS) and FS were not normally distributed with $\mathrm{p}$ values $<0.001,0.007$ and 0.026 , respectively. This suggests fitting the regression model using logarithm transformation of AF and original FS. The significant predictors with coefficients, their p-values and $R^{2}$ in the model are presented in Table 2.3. That is,

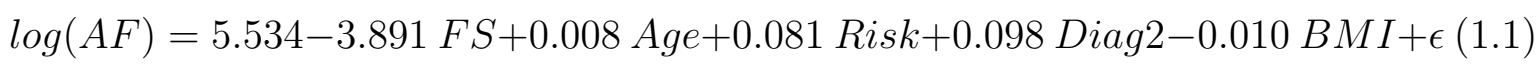

where Risk=1 for patient in AR group and 0 in NR group. Based on the regression model, the values of FS and the covariates, the 34 missing AF values are imputed. Thus, after imputing the missing values the total sample size is 272 .

Because AF is the only variable with missing values in our data $(n=272)$, we consider the data including age at diagnosis, BMI, diagnosis group, risk group, FS. Based on each imputation data, we calculate the Cumulative Incidence Function 
(CIF) for constant exponential model, two or three piecewise exponential models using the method. Then, the methods were applied to the imputed data sets for each group, AR and NR, and both groups combined. Then, based on the likelihood ratio test, the corresponding p-value for group effect for the variable AF is 0.014 (median of $\mathrm{m}=20$ imputations). On the other hand, the $\mathrm{p}$-value without imputation is 0.020 (Rai et al., 2013). The results with imputation seem to be little bit more sensitive for the group effect in AF compared to that without imputation. Note that not using the illness/death model as proposed and, instead, using logistic regression, without imputation, the group effect was only marginally significant $(p=0.065)$ (Hudson et al., 2007; Rai et al., 2013). Thus, the approach based on illness/death model led to a better understanding of this data and motivated the current development. A summary of the results is given in Table 2.4. The cumulative incidence function (CIF) was derived for exponential and piecewise exponential models for the imputed data using the above regressions model and SAS procedure (PROC MI, with $\mathrm{m}=5$, 10 and 100 imputations) when compared to those based on the original data (without imputation).

In Table 2.4, the group effects are reported for both data without imputation and with imputation for $\mathrm{m}=5,20$ and 100 . For imputed data, we reported the pvalues of group effect as mean, minimum, maximum, and median. A comparison of cumulative incidence rates can be found in Figure 2.3.

\subsubsection{Simulation Study}

To assess the performance of the imputation approach we conducted simulation studies as described below.

The primary focus is on assessing the performance of imputing AF in Anthracycline Cardiac Toxicity data for comparing the cumulative incidences of cardiac toxicity in the illness-death model as discussed above. The detailed steps are de- 
scribed as follows.

Step 1: From Table 2.3 it is clear that $\log (\mathrm{AF})$ values are associated with the risk group (AR and NR), diagnosis group (Sarcomas vs. others), Age, FS, and BMI and using the equation (1.1) we first filled all the missing values of AF with the mean predicted values and obtained a complete copy of the data set.

Step 2: Then, for simulation studies we first created four subgroups:

Group 1: The patients which are in AR group diagnosed with Sarcomas (sample size $\left.n_{1}\right)$

Group 2: The patients which are in AR group diagnosed with other cancers (sample size $\left.n_{2}\right)$.

Group 3: The patients which are in NR group diagnosed with Sarcomas (sample size $\left.n_{3}\right)$.

Group 4: The patients which are in NR group diagnosed with other cancers (sample size $\left.n_{4}\right)$.

Now to keep the covariance structure consistent with the observed data the sample mean and variance-covariance matrix were obtained for the variables $\mathrm{LAF}=\log (\mathrm{AF})$, FS, Age, BMI and LTime= $\log$ (Time), where Time is the length of follow-up from diagnosis to the time of survey and the individual can be in one of the three states as shown in Figure 1.1.

Step 3: (Generate multi-normal data by group): Generate a sequence of random vectors, $(L A F, F S, A g e, B M I, L T i m e)_{j}, j=1, \ldots, n_{i}$, from multi-normal distribution for Group $i(i=1,2,3,4)$. That is, we assumed that,

$(L A F, F S, \text { Age, BMI, LTime })_{j} \sim$ i.i.d. $M V N\left(v_{i}, \Sigma_{i}\right)$ for $j=1,2, \ldots, n_{i}, i=1,2, \ldots, 4$, 
where $n_{1}=n_{2}=n / 3$ and $n_{3}=n_{4}=n / 6$ for $n=180,240$, or 300 (the sample sizes for three simulation studies) and $v_{i}$ and $\Sigma_{i}$ are the sample mean vector and covariance matrix. In this simulation study we used unequal samples size to reflect higher proportion of At-risk survivors in our cohort.

Note: We noticed the means of FS and LAF were significantly low (high) for Group 1, which would have led to highly significant p-values for comparing the two groups (AR vs. NR). Therefore, we adjusted the mean values for these two variables in the simulation studies as follows:

\begin{tabular}{|l|c|c|c|c|c|}
\hline \multicolumn{5}{|c|}{ Mean of FS and LAF in Anthracycline Cardiac Toxicity data } \\
\hline \multirow{2}{*}{} & \multicolumn{4}{|c|}{ Group } \\
\cline { 2 - 6 } & & 1 & 2 & 3 & 4 \\
\hline \multirow{2}{*}{ Original } & FS & 0.307 & 0.342 & 0.359 & 0.358 \\
\cline { 2 - 6 } & LAF & 4.209 & 3.932 & 3.865 & 3.786 \\
\hline \multirow{2}{*}{ Adjusted } & FS & $\mathbf{0 . 3 3 0}$ & 0.342 & 0.359 & 0.358 \\
\cline { 2 - 6 } & LAF & $\mathbf{4 . 0 0 0}$ & 3.932 & 3.865 & 3.786 \\
\hline
\end{tabular}

Step 4: (Incomplete Data in AF): From the sample size generated in Step 2, we randomly deleted $\mathrm{R} \%$ ( $\mathrm{R}=20$ or 30$)$ of $A F$ values, and got incomplete data with sample sizes $(100-\mathrm{R}) \% \mathrm{n}$.

Step 5: (Imputed data): Using SAS procedure PROC MI with FCS option we imputed AF values and obtained a complete copy of the data set.

Step 6: (Calculate p-value for group effect): For the one parameter exponential distribution, the p-values for group effect (comparing AR with NR) were obtained using the likelihood ratio test for complete (originally generated), incomplete and imputed data set. 
Step 7: The imputation process (Step 5) was repeated 20 times to obtain 20 copies of complete data sets, which resulted in 20 p-values. A description of the p-values in terms of mean, median, minimum, and maximum is summarized in Tables 2.5-2.7.

Step 8: Steps $2-7$ were repeated 10 times to assess the performance of the imputation approach on 10 independently generated data sets. Simulation results are presented in Tables 2.5-2.7.

From Tables 2.5-2.7, it is seen that, in general, the median of the p-values is much closer to the p-value obtained from the complete data compared to those obtained from the incomplete data. However, there are some extreme situations where the results from the complete data and those obtained from imputations and incomplete data are not in agreement and this could be due to chance that more observations were deleted from a particular group and the regression is not able to completely exploit the underlying correlation structure. For example, for the $4^{\text {th }}$ simulation, when the sample size is 180 and $30 \%$ of the observations are imputed the p-value for the complete data set is 0.007 , but those corresponding to incomplete data and imputed data were 0.059 and 0.469 , respectively. This clearly suggests that the manner in which data are generated and the observations are randomly deleted might have changed the underlying structure particularly those who are in the AR and NR groups. However, for lager sample size $(n=300)$ we see that in all simulations the median p-value based on imputation is much closer to the p-value obtained from the complete data. Thus, it is clear that with imputation approach we are able to exploit the underlying correlation structure and obtain nearly unbiased conclusions. 


\subsection{Discussion}

In this paper, we have employed the illness/death model developed in Rai et al. (2013) and demonstrated an example by imputing the missing observations in a Phase IV current status data. Though the methodology has been applied on a parametric model, it can be easily extended to other models under different settings. Clinical data are usually analyzed using meanly log rank test or Kaplan Meier curves due to its simplicity (Kaplan and Meier, 1958). However, in the setting of interval censored data the approach proposed here is simple to use and can be implemented easily to estimate fixed-time cumulative incidence function with or without imputation (Rai et al., 2013).

In order to monitor the long-term effects of the cancer treatment, the longitudinally followed patients are evaluated multiple times throughout their adulthood. At every visit multiple health indicators are measured and recorded. Besides from cardiotoxicity, other events such as obesity and secondary cancer might have occurred. Some of these events can be identified as competing risk while other events might be correlated to the variables we are interested in. This leads to multivariate time-toevent data. In our example, cardiotoxicity can be determined by looking at abnormal $\mathrm{AF}$ and FS values, but there are some other measures to evaluate cardiotoxicity such as systemic vascular resistance (SVR). For some reasons, not all patients had both measures. Based on bi-variate assumption, the model would only include subjects that have valid information on both outcomes and this would reduce the sample size and potentially ignore important information. Based on this consideration, the multivariable regression method was used to impute the missing observations and to apply the parametric method to the imputed data and compare the results with those obtained without imputation.

As stated before, the problem of evaluating possible toxic effects of cancer ther- 
apies in a Phase IV trial setting is an important problem. Among the many issues in such studies, missing data is a key aspect that can influence the inferences. It is also important to understand the nature of impact of missing data on the analysis and the interpretation of the study data. Also note that imputation increases the sample size, and thus increases statistical power to detect the same effect size, but if the model assumptions are not correct, the inference may not be valid. Thus, it is recommended to report the p-values with and without imputation. However, with higher absolute correlations between two outcome measures (the primary outcome measure, AF, with higher missing and the secondary outcome measure, FS, with little or no missing), produced efficient results, a rigorous simulation study with different amount of correlations between two outcome measures, amount of missing and model uncertainty is underway to consider this aspect and will be reported elsewhere; this is along the lines for our work of a randomized clinical study (Liu and De, 2015).

Another limitation of this study is that, this is a cross-sectional survey to estimate the long-term effect of cardiotoxicity of the primary treatment of cancer. Dodge (2003) defined cross-sectional survey "A method of data collection whereby a battery of questions is asked of participation at one single point or in a relatively small interval of time. Inferences about a population must be anchored to the time period in which the sample was taken. Data from cross-sectional surveys are typically unable to be used to prove the existence of cause-and-effect relationships." Even though, this is based on enrolling consecutive eligible patients in a very homogeneous environment (St. Jude Children's Hospital treats patients without charge to patients), the effect of this limitation is minimized, but cannot be reduced to zero. Generalization of the results to a general population should be done with caution. 


\subsection{Tables and Figures}

Figure 2.1: Scatter plot of AF and FS within AR and NR group of patients. Complete data (labeled c) displays strong correlation between AF and FS. Missing values of AF are in almost the entire range of FS values (labeled $\mathrm{m}$ ).

\section{AF Missing Pattern by Group}

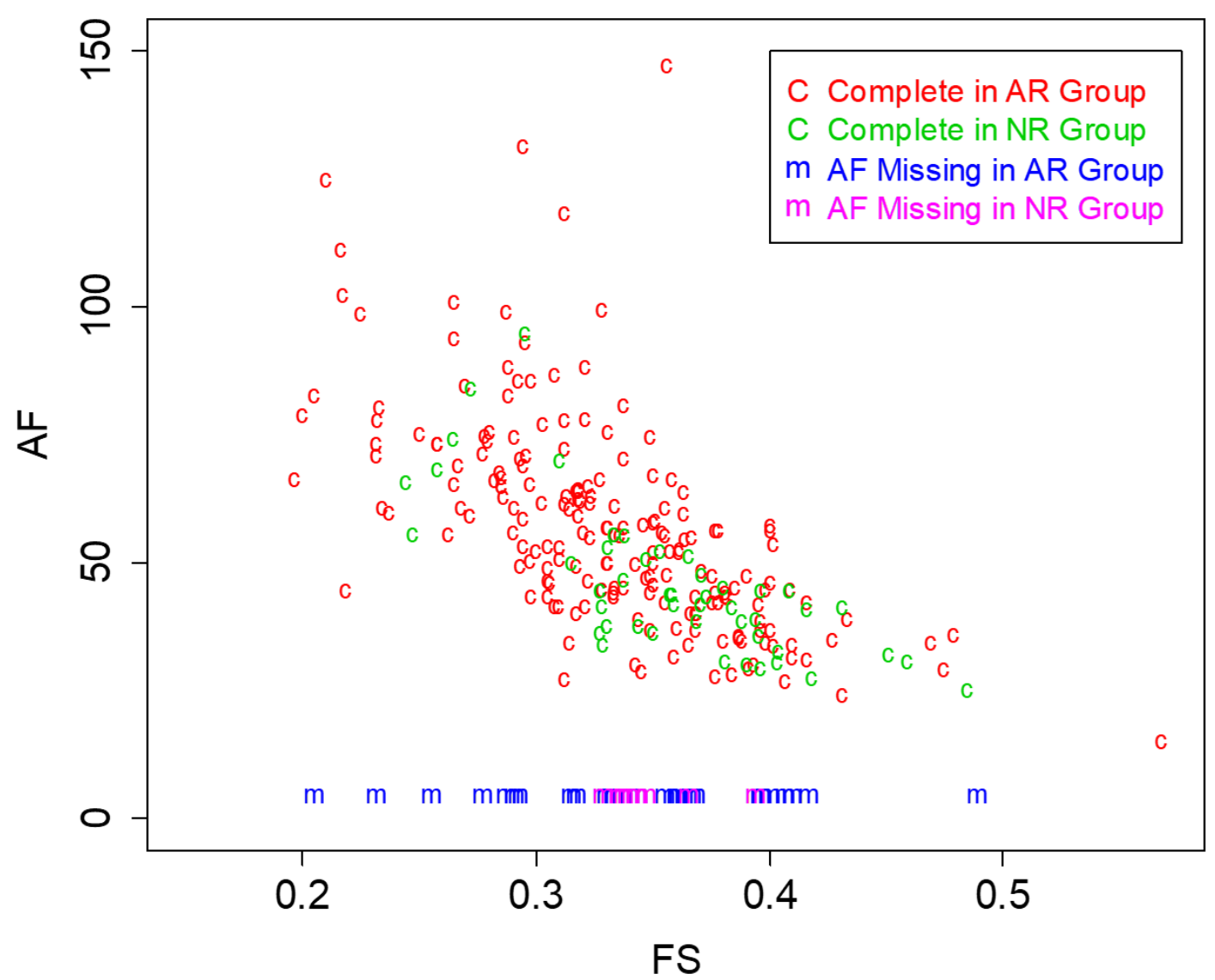


Figure 2.2: Piecewise Incidence Rate.

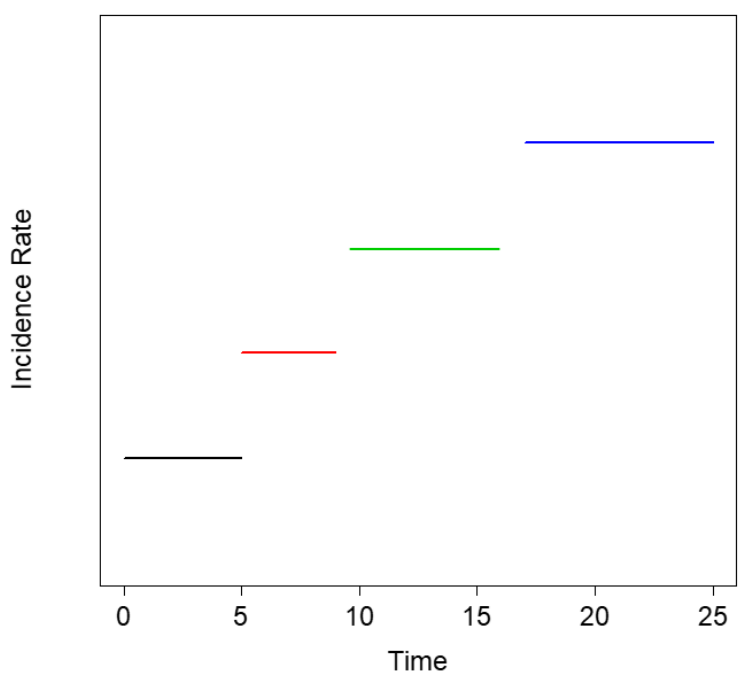


Figure 2.3: Cumulative Incidence Comparison for AF Based on Original Data and Imputed Data (Using the mean of intensity estimates) Corresponding to $\mathrm{m}=5,20$ and 100. Note: The lines for $m=5$ and 20 are almost overlapped.
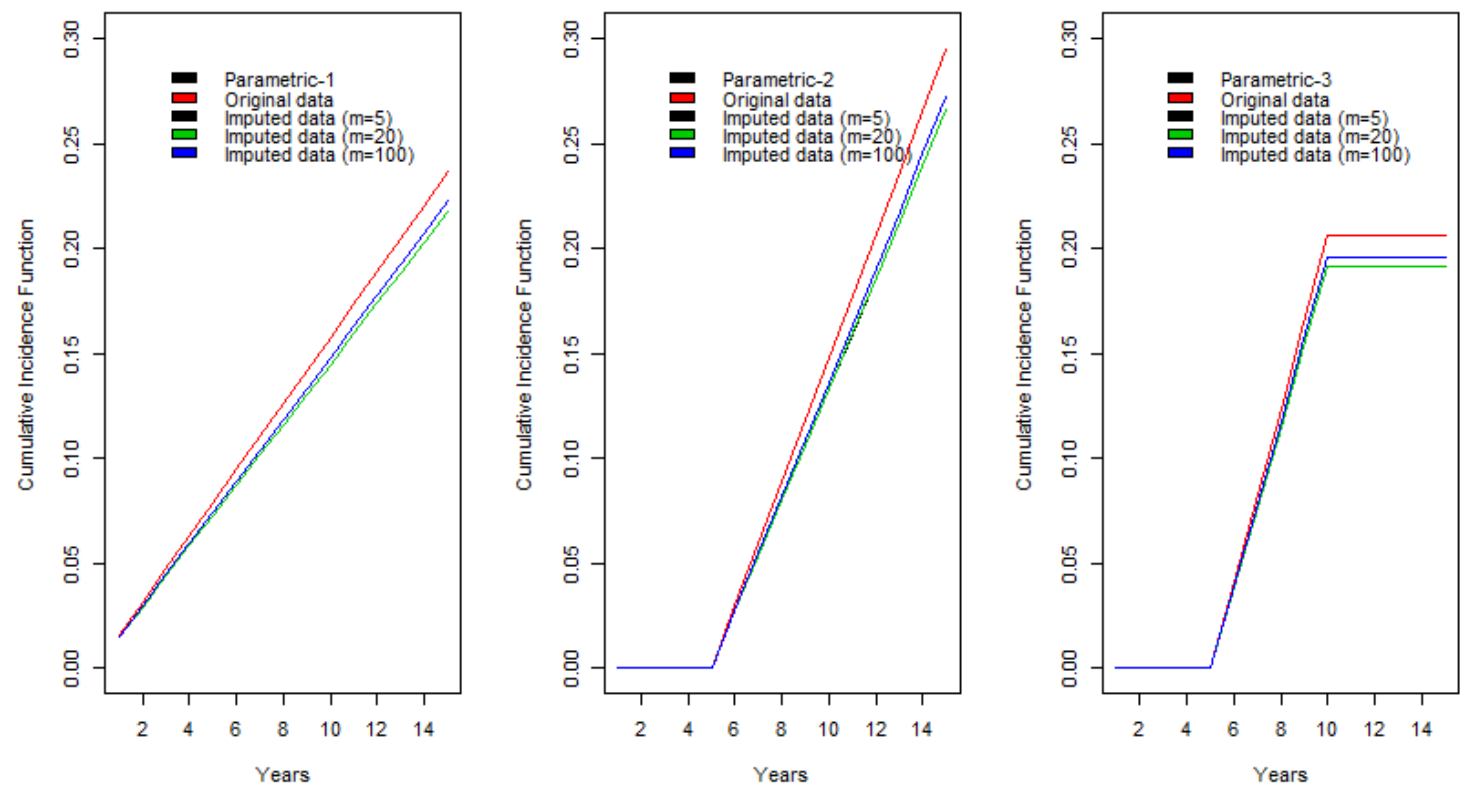
Table 2.1: Statistical Summary of the Patient Population in the Cardiac Study.

\begin{tabular}{|c|c|c|c|c|c|c|}
\hline \multirow[b]{2}{*}{ Demographics } & \multicolumn{2}{|c|}{$\begin{array}{l}\text { At-Risk Group } \\
\qquad(\mathrm{n}=\mathbf{2 2 3})\end{array}$} & \multicolumn{2}{|c|}{$\begin{array}{c}\text { Not At-Risk } \\
\text { Group } \\
(n=55)\end{array}$} & \multicolumn{2}{|c|}{$\begin{array}{c}\text { Total } \\
(n=278)\end{array}$} \\
\hline & $\mathbf{N}$ & $\%$ & $\mathbf{N}$ & $\%$ & $\mathbf{N}$ & $\%$ \\
\hline \multicolumn{7}{|l|}{ Sex } \\
\hline Male & 108 & 51.6 & 31 & 56.4 & 139 & 50 \\
\hline Female & 115 & 48.4 & 24 & 43.6 & 139 & 50 \\
\hline \multicolumn{7}{|l|}{ Treatment group } \\
\hline Anthracycline & 157 & 70.4 & 0 & 0 & 157 & 56.5 \\
\hline Anthracycline + Radiation & 60 & 26.9 & 0 & 0 & 60 & 21.6 \\
\hline Radiation & 6 & 2.7 & 0 & 0 & 6 & 2.1 \\
\hline None & 0 & 0 & 55 & 100 & 55 & 19.8 \\
\hline \multicolumn{7}{|l|}{ Race/ethnicity } \\
\hline White & 183 & 82.1 & 44 & 80.0 & 227 & 81.7 \\
\hline Black & 30 & 13.5 & 11 & 20.0 & 41 & 14.7 \\
\hline Other & 10 & 4.4 & 0 & 0 & 10 & 3.6 \\
\hline \multicolumn{7}{|l|}{ Diagnosis } \\
\hline Leukemia & 67 & 30.0 & 10 & 18.2 & 77 & 27.7 \\
\hline Sarcomas & 60 & 26.9 & 14 & 25.4 & 74 & 26.6 \\
\hline Lymphoma & 54 & 34.2 & 2 & 3.6 & 56 & 20.1 \\
\hline Embryonal tumors & 42 & 18.8 & 29 & 52.7 & 71 & 25.6 \\
\hline \multicolumn{7}{|c|}{ Age at Cancer Diagnosis, Years } \\
\hline $\mathrm{N}$ & \multicolumn{2}{|c|}{223} & \multicolumn{2}{|c|}{55} & \multicolumn{2}{|c|}{278} \\
\hline Mean & \multicolumn{2}{|c|}{7.37} & \multicolumn{2}{|c|}{5.77} & \multicolumn{2}{|c|}{7.05} \\
\hline Median & \multicolumn{2}{|c|}{5.46} & \multicolumn{2}{|c|}{3.11} & \multicolumn{2}{|c|}{4.68} \\
\hline Range & \multicolumn{2}{|c|}{$0.01-23.56$} & \multicolumn{2}{|c|}{$0.29-20.06$} & \multicolumn{2}{|c|}{$0.01-23.56$} \\
\hline \multicolumn{7}{|l|}{ Afterload } \\
\hline $\mathrm{N}$ & \multicolumn{2}{|c|}{191} & \multicolumn{2}{|c|}{47} & \multicolumn{2}{|c|}{238} \\
\hline Mean & \multicolumn{2}{|c|}{57.50} & \multicolumn{2}{|c|}{45.73} & \multicolumn{2}{|c|}{55.18} \\
\hline Median & \multicolumn{2}{|c|}{55.43} & & & & \\
\hline Range & $15.38-$ & 7.32 & 25.6 & 5.02 & 15.38 & 47.32 \\
\hline Fractional Shortening & & & & & & \\
\hline $\mathrm{N}$ & 21 & & & & & \\
\hline Mean & 0.3 & & & & & \\
\hline Median & 0.3 & & & & & \\
\hline Range & 0.20 & & & .49 & 0.2 & .57 \\
\hline
\end{tabular}


Table 2.2: Likelihood Contributions Corresponding to Different Observation Types.

\begin{tabular}{|l|l|l|}
\hline \multicolumn{1}{|c|}{ Observation Type } & \multicolumn{1}{|c|}{ Outcome } & \multicolumn{1}{c|}{ Likelihood Contribution } \\
\hline $\begin{array}{l}\text { Death with } \\
\text { No Cardiac } \\
\text { Abnormality }\end{array}$ & $T=t, X\left(t^{-}\right)=1$ & $L_{1}(t)=\lambda_{2}(t) Q(t)$ \\
\hline $\begin{array}{l}\text { Alive with } \\
\text { No Cardiac } \\
\text { Abnormality }\end{array}$ & $T>t, X(t)=1$ & $L_{2}(t)=Q(t)$ \\
\hline $\begin{array}{l}\text { Death/Cardiac Failure } \\
\text { with Cardiac } \\
\text { Abnormality }\end{array}$ & $T=t, X\left(t^{-}\right)=2$ & $L_{3}(t)=\int_{0}^{t} \lambda_{1}(u) Q(u) \lambda_{3}(t \mid u) Q_{3}(t \mid u) d u$ \\
\hline $\begin{array}{l}\text { Alive with } \\
\text { Cardiac Abnormality }\end{array}$ & $T>t, X(t)=2$ & $L_{4}(t)=\int_{0}^{t} \lambda_{1}(u) Q(u) Q_{3}(t \mid u) d u$ \\
\hline
\end{tabular}


Table 2.3: Coefficients and p-values in Regression Model.

\begin{tabular}{|l|c|c|c|}
\hline \multicolumn{1}{|c|}{ Variable } & Estimator & SE & p-value \\
\hline Intercept & 5.534 & 0.106 & $<0.001$ \\
\hline FS & -3.891 & 0.274 & $<0.001$ \\
\hline $\begin{array}{l}\text { Age at } \\
\text { Diagnosis }\end{array}$ & 0.008 & 0.003 & 0.005 \\
\hline Risk Group & 0.081 & 0.038 & 0.033 \\
\hline Diag2 & 0.098 & 0.035 & 0.006 \\
\hline BMI & -0.010 & 0.003 & $<0.001$ \\
\hline$R^{2}$ & \multicolumn{3}{|l}{0.586} \\
\hline
\end{tabular}


Table 2.4: AF p-values for Group Effect using Likelihood Ratio Test.

\begin{tabular}{|c|c|c|c|c|c|}
\hline \multirow{2}{*}{ Procedure } & \multirow{2}{*}{$\begin{array}{l}\text { Without } \\
\text { Imputation }\end{array}$} & \multicolumn{4}{|c|}{ With Imputation (MI) } \\
\hline & & mean & median & $\min$ & $\max$ \\
\hline \multicolumn{6}{|l|}{ Parameter-1 } \\
\hline $\mathrm{m}=5$ & \multirow{3}{*}{0.0199} & 0.0125 & 0.0121 & 0.0098 & 0.0187 \\
\hline $\mathrm{m}=20$ & & 0.0180 & 0.0138 & 0.0098 & 0.0561 \\
\hline $\mathrm{m}=100$ & & 0.0160 & 0.0123 & 0.0051 & 0.0696 \\
\hline \multicolumn{6}{|l|}{ Parameter-2 } \\
\hline$m=5$ & \multirow{3}{*}{0.0118} & 0.0082 & 0.0077 & 0.0063 & 0.0126 \\
\hline $\mathrm{m}=20$ & & 0.0120 & 0.0091 & 0.0063 & 0.0397 \\
\hline $\mathrm{m}=100$ & & 0.0105 & 0.0080 & 0.0031 & 0.0487 \\
\hline \multicolumn{6}{|l|}{ Parameter-3 } \\
\hline $\mathrm{m}=5$ & \multirow{3}{*}{0.0782} & 0.0564 & 0.0519 & 0.0469 & 0.0805 \\
\hline $\mathrm{m}=20$ & & 0.0743 & 0.0621 & 0.0469 & 0.1983 \\
\hline $\mathrm{m}=100$ & & 0.0656 & 0.0565 & 0.0206 & 0.2164 \\
\hline
\end{tabular}


Table 2.5: p-values for Group Effect using Likelihood Ratio Test ( $\mathrm{n}=180)$.

\begin{tabular}{|c|c|c|c|c|c|c|c|c|c|}
\hline & & & Complete & Incomplete & \multicolumn{5}{|c|}{ Imputed Data (p-values) } \\
\hline $\mathrm{n}$ & $\mathrm{R} \%$ & $\begin{array}{c}\text { Simulation } \\
\#\end{array}$ & p-value & $\mathrm{p}$-value & Median & Min & Max & Mean & SD \\
\hline \multirow{10}{*}{180} & \multirow{10}{*}{20} & 1 & $<.001$ & $<.001$ & $<.001$ & $<.001$ & 0.011 & 0.001 & 0.003 \\
\hline & & 2 & 0.285 & 0.322 & 0.256 & 0.127 & 0.628 & 0.300 & 0.143 \\
\hline & & 3 & 0.004 & 0.019 & 0.006 & 0.002 & 0.014 & 0.006 & 0.003 \\
\hline & & 4 & 0.007 & 0.057 & 0.025 & 0.003 & 0.202 & 0.045 & 0.050 \\
\hline & & 5 & 0.003 & 0.003 & 0.004 & $<.001$ & 0.025 & 0.007 & 0.008 \\
\hline & & 6 & $<.001$ & 0.001 & 0.005 & $<.001$ & 0.073 & 0.011 & 0.016 \\
\hline & & 7 & 0.013 & 0.031 & 0.020 & 0.002 & 0.088 & 0.027 & 0.027 \\
\hline & & 8 & 0.761 & 0.937 & 0.770 & 0.539 & 0.995 & 0.801 & 0.135 \\
\hline & & 9 & 0.007 & 0.001 & $<.001$ & $<.001$ & 0.100 & 0.008 & 0.024 \\
\hline & & 10 & 0.011 & 0.099 & 0.026 & 0.004 & 0.191 & 0.045 & 0.053 \\
\hline & & & & & & & & & \\
\hline \multirow{10}{*}{180} & \multirow{10}{*}{30} & 1 & $<.001$ & 0.130 & 0.004 & $<.001$ & 0.091 & 0.015 & 0.027 \\
\hline & & 2 & 0.285 & 0.002 & 0.035 & 0.005 & 0.130 & 0.046 & 0.037 \\
\hline & & 3 & 0.004 & 0.003 & $<.001$ & $<.001$ & 0.003 & $<.001$ & 0.001 \\
\hline & & 4 & 0.007 & 0.059 & 0.469 & 0.115 & 0.889 & 0.493 & 0.230 \\
\hline & & 5 & 0.003 & 0.033 & 0.314 & 0.022 & 0.935 & 0.330 & 0.253 \\
\hline & & 6 & $<.001$ & 0.040 & 0.001 & $<.001$ & 0.060 & 0.007 & 0.015 \\
\hline & & 7 & 0.013 & 0.262 & 0.008 & $<.001$ & 0.090 & 0.020 & 0.026 \\
\hline & & 8 & 0.761 & 0.003 & 0.035 & 0.003 & 0.150 & 0.050 & 0.044 \\
\hline & & 9 & 0.007 & 0.005 & $<.001$ & $<.001$ & 0.032 & 0.005 & 0.009 \\
\hline & & 10 & 0.011 & 0.250 & 0.140 & 0.011 & 0.372 & 0.140 & 0.100 \\
\hline
\end{tabular}


Table 2.6: p-values for Group Effect using Likelihood Ratio Test $(\mathrm{n}=240)$.

\begin{tabular}{|c|c|c|c|c|c|c|c|c|c|}
\hline & & & Complete & $\begin{array}{l}\text { Incomplete } \\
\text { Data }\end{array}$ & \multicolumn{5}{|c|}{ Imputed Data (p-values) } \\
\hline $\mathrm{n}$ & $\mathrm{R} \%$ & $\begin{array}{c}\text { Simulation } \\
\#\end{array}$ & p-value & p-value & Median & Min & Max & Mean & SD \\
\hline \multirow{10}{*}{240} & \multirow{10}{*}{20} & 1 & $<.001$ & $<.001$ & $<.001$ & $<.001$ & 0.003 & $<.001$ & 0.001 \\
\hline & & 2 & 0.087 & 0.075 & 0.087 & 0.011 & 0.248 & 0.094 & 0.072 \\
\hline & & 3 & 0.020 & 0.041 & 0.040 & 0.003 & 0.117 & 0.043 & 0.026 \\
\hline & & 4 & 0.011 & 0.005 & 0.007 & $<.001$ & 0.086 & 0.017 & 0.022 \\
\hline & & 5 & 0.002 & 0.016 & 0.009 & 0.001 & 0.74 & 0.025 & 0.060 \\
\hline & & 6 & $<.001$ & $<.001$ & $<.001$ & $<.001$ & 0.001 & $<.001$ & $<.001$ \\
\hline & & 7 & 0.001 & 0.001 & 0.001 & $<.001$ & 0.008 & 0.001 & 0.002 \\
\hline & & 8 & 0.221 & 0.390 & 0.347 & 0.109 & 0.933 & 0.388 & 0.197 \\
\hline & & 9 & 0.001 & $<.001$ & $<.001$ & $<.001$ & 0.003 & 0.001 & 0.001 \\
\hline & & 10 & $<.001$ & 0.001 & $<.001$ & $<.001$ & 0.033 & 0.002 & 0.007 \\
\hline & & & & & & & & & \\
\hline \multirow{10}{*}{240} & \multirow{10}{*}{30} & 1 & $<.001$ & $<.001$ & $<.001$ & $<.001$ & $<.001$ & $<.001$ & $<.001$ \\
\hline & & 2 & 0.087 & 0.327 & 0.105 & 0.102 & 0.499 & 0.154 & 0.141 \\
\hline & & 3 & 0.020 & 0.060 & 0.023 & 0.004 & 0.135 & 0.037 & 0.038 \\
\hline & & 4 & 0.011 & 0.028 & 0.011 & 0.001 & 0.140 & 0.027 & 0.033 \\
\hline & & 5 & 0.002 & 0.014 & 0.008 & $<.001$ & 0.042 & 0.011 & 0.013 \\
\hline & & 6 & $<.001$ & $<.001$ & $<.001$ & $<.001$ & 0.004 & 0.001 & 0.001 \\
\hline & & 7 & 0.001 & 0.002 & 0.001 & $<.001$ & 0.081 & 0.011 & 0.022 \\
\hline & & 8 & 0.221 & 0.121 & 0.041 & 0.004 & 0.201 & 0.051 & 0.047 \\
\hline & & 9 & 0.001 & 0.019 & 0.012 & 0.001 & 0.079 & 0.022 & 0.026 \\
\hline & & 10 & $<.001$ & 0.005 & 0.015 & $<.001$ & 0.159 & 0.041 & 0.054 \\
\hline
\end{tabular}


Table 2.7: p-values for Group Effect using Likelihood Ratio Test ( $\mathrm{n}=300)$.

\begin{tabular}{|c|c|c|c|c|c|c|c|c|c|}
\hline & & & $\begin{array}{c}\text { Complete } \\
\text { Data }\end{array}$ & $\begin{array}{c}\text { Incomplete } \\
\text { Data }\end{array}$ & \multicolumn{5}{|c|}{ Imputed Data (p-values) } \\
\hline $\mathrm{n}$ & $\mathrm{R} \%$ & $\begin{array}{c}\text { Simulation } \\
\#\end{array}$ & p-value & p-value & Median & Min & Max & Mean & SD \\
\hline \multirow{10}{*}{300} & \multirow{10}{*}{20} & 1 & $<.001$ & $<.001$ & $<.001$ & $<.001$ & 0.001 & $<.001$ & $<.001$ \\
\hline & & 2 & 0.268 & 0.107 & 0.155 & 0.023 & 0.809 & 0.212 & 0.184 \\
\hline & & 3 & 0.026 & 0.011 & 0.014 & 0.001 & 0.077 & 0.022 & 0.022 \\
\hline & & 4 & $<.001$ & $<.001$ & $<.001$ & $<.001$ & 0.003 & $<.001$ & 0.001 \\
\hline & & 5 & $<.001$ & 0.001 & 0.001 & $<.001$ & 0.005 & 0.001 & 0.002 \\
\hline & & 6 & $<.001$ & 0.028 & 0.007 & 0.001 & 0.056 & 0.014 & 0.015 \\
\hline & & 7 & 0.001 & 0.003 & 0.002 & $<.001$ & 0.016 & 0.004 & 0.005 \\
\hline & & 8 & 0.107 & 0.193 & 0.118 & 0.031 & 0.370 & 0.148 & 0.098 \\
\hline & & 9 & $<.001$ & 0.001 & 0.002 & $<.001$ & 0.017 & 0.004 & 0.005 \\
\hline & & 10 & 0.001 & 0.013 & 0.007 & 0.001 & 0.336 & 0.047 & 0.090 \\
\hline & & & & & & & & & \\
\hline \multirow{10}{*}{300} & \multirow{10}{*}{30} & 1 & $<.001$ & $<.001$ & $<.001$ & $<.001$ & $<.001$ & $<.001$ & $<.001$ \\
\hline & & 2 & 0.268 & 0.759 & 0.383 & 0.078 & 0.889 & 0.389 & 0.231 \\
\hline & & 3 & 0.026 & 0.024 & 0.019 & 0.003 & 0.103 & 0.032 & 0.026 \\
\hline & & 4 & $<.001$ & 0.003 & $<.001$ & $<.001$ & 0.007 & 0.001 & 0.002 \\
\hline & & 5 & $<.001$ & 0.009 & 0.001 & $<.001$ & 0.056 & 0.006 & 0.013 \\
\hline & & 6 & $<.001$ & 0.015 & 0.003 & $<.001$ & 0.042 & 0.009 & 0.014 \\
\hline & & 7 & 0.001 & 0.015 & 0.003 & $<.01$ & 0.022 & 0.002 & 0.006 \\
\hline & & 8 & 0.107 & 0.169 & 0.110 & 0.007 & 0.620 & 0.142 & 0.142 \\
\hline & & 9 & $<.001$ & $<.001$ & $<.001$ & $<.001$ & 0.003 & $<.001$ & 0.001 \\
\hline & & 10 & 0.001 & 0.002 & 0.003 & $<.001$ & 0.114 & 0.015 & 0.027 \\
\hline
\end{tabular}




\section{CHAPTER 3 \\ ESTIMATING TRANSITION INTENSITY RATE ON INTERVAL CENSORED DATA USING SEMI-PARAMETRIC WITH EM ALGORITHM APPROACH}

\subsection{Introduction}

\subsubsection{Background}

Cancer is among one of the leading causes of death in children. In fact, it is the second leading cause of death just after accidents in children ages 1 to 14 . According to the American Cancer Society, approximately 10,500 children under the age of 15 will be diagnosed with cancer in 2021, and that number is just in the United States alone. Globally more than 400,000 children are diagnosed with cancer every year. Though childhood cancer rates have been rising for the past few decades, fortunately, with significant advancements in treatment, survival rates have dramatically increased. Recent statistics showed that the 5-year survival rate for children is $84 \%$ compared to only $58 \%$ in the mid-70s (American Cancer Society). However, this encouraging news comes with some disappointment, that is, childhood cancer survivors are often at a higher risk of developing late adverse effects, or even mortality, during their adulthood. It is unfortunate that these children who have already suffered through treatments in their childhood may encounter more suffering as long term sequalae to their cancer or its treatment. With the increasing number of childhood cancer survivors worldwide, monitoring for long-term treatment effects is crucial. In 
that context Phase IV clinical trials, such as St. Jude Lifetime Cohort Study, play an important role in understanding the extent of the long-term complications of the treatment effect and the risk factors associated with them. Additionally, the insight gained from these studies could provide valuable information that could be used to improve the future treatment plans.

Among childhood cancer survivors, cardiovascular events are among the top nonmalignant causes of death (Armstrong et al., 2009). This is due to the damage to cardiomyocytes caused by chemotherapy and chest radiation therapy received during the cancer treatment (Lipshultz et al., 2013). Even though certain chemotherapy such as anthracycline are well known for its cardiotoxic (Hudson et al., 2007), it remains a regularly used component of the treatment because of its therapeutic benefits. It is important to mention that cardiotoxicity is irreversible but preventable. However, the timing of the intervention is crucial because the therapy itself may be toxic. This motivates us to estimate the cumulative incidence of the cardiotoxicity in childhood cancer survivors because such information could be used to help researchers identify the best time to intervene. More information regarding the causes, treatment, and prevention of cardiotoxicity can be found in a comprehensive review by Koutsoukis et al. (2018).

Since it is not practical to follow the survivor continuously in real time in the long-term follow-up studies because of financial and logistical reasons, the exact time of cardiotoxicity is, often, not known. However, health related information is obtained through cross-sectional surveys or when survivors came back to the hospital and get evaluated periodically, in a fixed time interval (every month or every year) or in an ad hoc manner. Although the exact time of cardiotoxicity is unknown, the current status information is available and from there we know that the cardiotoxicity occurred sometime in the interval between the two follow-up visits. Such dataset can be characterized as case I interval censored data (Sun, 2006; Rai, 2008). 


\subsubsection{Methodology Review}

The analysis of interval censored data has been widely discussed. Ayer et al. (1955) and van Eeden $(1956,1957)$ first introduced the nonparametric procedures for deriving maximum likelihood estimators. More recently, Sun (2006) discussed a more comprehensive way of analyzing interval censored data using a nonparametric approach. In semiparametric settings, Huang (1995, 1996) discussed and established the normality of maximum likelihood estimator. More recently, Goetghebeur and Ryan (2000) proposed a semiparametric approach to the proportional hazards regression analysis of interval censored data. In terms of parametric settings, Rai et al. (2013) first introduced a piecewise exponential model for handling current status data. Later, Srivastava et al. (2018) proposed using Weibull distribution to overcome the limitation that existed in the piecewise exponential and found it to be more robust. Pradhan and Kundu (2014) also used Weibull distribution as the underlying lifetime distribution for the interval censored data, but suggested using EM algorithm approach.

The prevalence of events can be estimated easily from the interval censored data, but that does not provide useful information. Rather, the incidence rate should be calculated since every subject is followed with different lengths of time. Both Rai et al. (2013) and Srivastava et al. (2018) used parametric approach with the assumption that the transition intensity rates from different risk groups are independent. It is true that subjects in different groups are independent but the rates can be modeled as dependent in certain way. In other words, the parametric approach may not be suitable if the underlying parametric assumptions do not hold. We have the same interest as Rai et al. (2013) and Srivastava et al. (2018), but focusing on using a semi-parametric approach with EM algorithm. Rai and Matthews (1997) introduced 
the discrete scale models for estimating transition intensity rate in a survival-sacrifice experiment using EM algorithm. Later, Rai, Matthews and Krewski (2000) extended the similar methodology to a mixed-scale model with an EM algorithm approach as well.

EM algorithm is a powerful procedure to use when no closed form solution can be obtained from the likelihood function. Besides from the traditional EM algorithm, Rai and Matthews (1993) introduced a modified approach that could potentially save time (less iterations). A detailed application demonstration of EM algorithm can be found in Gunaratnam and Rai (2019). Additionally, when dealing with highdimensional parameters, regular simple case likelihood function will not work. Rather, the use of profile likelihood should be considered (Murphy and Van Der Vaart, 2000). The details of the approach will be discussed in the next few sections.

\subsubsection{Motivating Example}

This research was motivated by St. Jude Lifetime Cohort Study (Hudson et al., 2011). The study was initiated in 2007 and is an ongoing retrospective cohort with prospective follow-up, which can be classified as a Phase IV study. The objective of the study is to establish a cohort of childhood cancer survivors, who were treated at St. Jude and have survived 5 years or more after cancer diagnosis, to better understand the long term sequalae of the cancer and its treatment. SJLIFE participants fill our detailed questionnaires and undergo comprehensive medical evaluations on a regular basis. Due to the nature of the study the patient can enter the cohort with highly variable length of follow-up from cancer diagnosis and the follow-up times after entering the cohort also may be highly variable. One of the long-term consequences of cancer patients treated with cardiotoxic therapy (treated with anthracycline and/or chest radiation) are at a very risk of developing cardiac abnormalities such as abnor- 
mal EF (Ejection Fraction) or abnormal SVR (Systemic Vascular Resistance). Let us denote this group as AR (At Risk group), and let NR represent the groups of survivors who were not treated with cardiotoxic therapy. Within this context it would be important to know the timing of the onset of these cardiotoxicities so that timely interventions could be implemented. That is, we are interested in estimating the CI of the cardiac events. However, given that the data on cardiotoxicity is collected intermittently the occurrence of the events falls in the category of interval censored data discussed above. The focus of this manuscript is to develop a statistical approach that would be able to estimate the CI of cardiac abnormality using interval censored data. The purpose of using a SJLIFE cohort is to illustrate the usefulness of the proposed methodology in estimating the CI and should not be used to draw any clinical conclusions. To better understand the problem at hand, let us define Year 1 as 5 years after treatment completion and Year 2 as 6 years after treatment completion, a patient can choose to participate in the study either at Year 1 or Year 2 , or even later. The challenge comes when we build the likelihood functions that will be discussed in the next section.

To help better understand the process, the following table is created to reflect the data characteristics in a complete data setting. The following table would corespond to survivors who have come for SJLIFE evaluations for the first time and the maximum follow-up time is 8 years afterr cohort study. 


\begin{tabular}{|c|c|c|c|c|c|c|c|c|c|}
\hline Year & $r_{+1}$ & $r_{+2}$ & $r_{+3}$ & $r_{+4}$ & $r_{+5}$ & $r_{+6}$ & $r_{+7}$ & $r_{+8}$ & Rate \\
\hline 1 & $n_{11}$ & $n_{12}$ & $n_{13}$ & $n_{14}$ & $n_{15}$ & $n_{16}$ & $n_{17}$ & $n_{18}$ & $\lambda_{11}$ \\
\hline 2 & & $n_{22}$ & $n_{23}$ & $n_{24}$ & $n_{25}$ & $n_{26}$ & $n_{27}$ & $n_{28}$ & $\lambda_{12}$ \\
\hline 3 & & & $n_{33}$ & $n_{34}$ & $n_{35}$ & $n_{36}$ & $n_{37}$ & $n_{38}$ & $\lambda_{13}$ \\
\hline 4 & & & & $n_{44}$ & $n_{45}$ & $n_{46}$ & $n_{47}$ & $n_{48}$ & $\lambda_{14}$ \\
\hline 5 & & & & & $n_{55}$ & $n_{56}$ & $n_{57}$ & $n_{58}$ & $\lambda_{15}$ \\
\hline 6 & & & & & & $n_{66}$ & $n_{67}$ & $n_{68}$ & $\lambda_{16}$ \\
\hline 7 & & & & & & & $n_{77}$ & $n_{78}$ & $\lambda_{17}$ \\
\hline 8 & & & & & & & & $n_{88}$ & $\lambda_{18}$ \\
\hline
\end{tabular}

It is assumed that we are interested in looking at intensity rates up to eight years. Let $\lambda_{1 i}, i=1,2, \ldots, 8$ be the intensity rates at every year (Year 1 , Year 2, etc.). Let $r_{+i}, i=1,2, \ldots, 8$ be the number of subjects that come to the clinic every year. For example, $r_{+1}$ patients come to the clinic with 1 year of followup, and $r_{+2}$ patients come to the clinic with 2 years of followup. Within each year, there are $n$ events (patients are abnormal). However, when a patient comes at Year 2, he/she may become abnormal either in Year 1 or in Year 2, but that information is unknown. Therefore, we define $n_{12}+n_{22}$ as the total number of events in Year 2 in which $n_{12}$ represent the events that occurred during Year 1 and $n_{22}$ represent the events that occurred during Year 2.

\subsection{Method}

\subsubsection{Overall Flow of the Analysis}

We prepared the following flow chart to clearly show the steps of the analysis. 


\section{Flow of the Analysis}

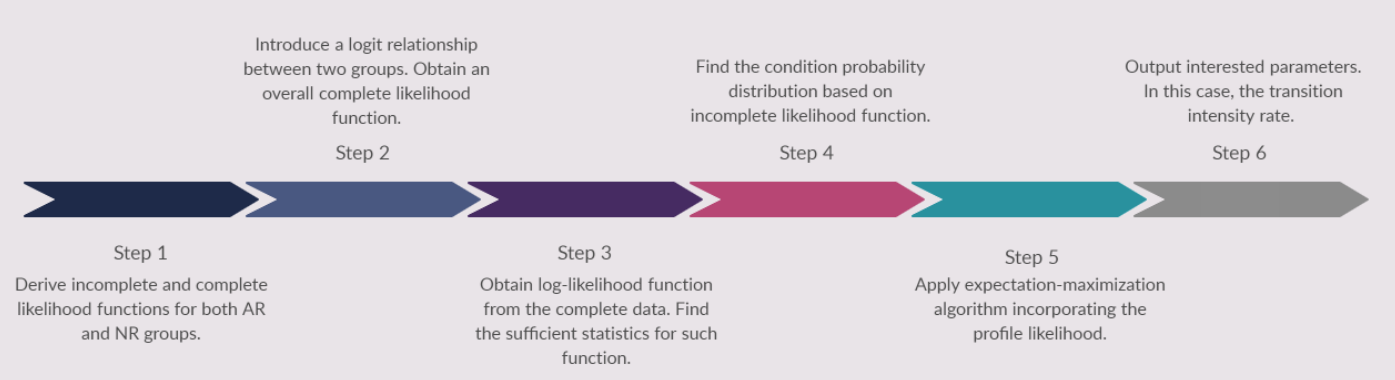

We now carry out the detailed steps in the next section as illustrated in the chart above.

\subsubsection{Detailed Steps of the Analysis}

The likelihood function should include all characteristics of the data set. This is somehow challenging in our situation. Here we present a likelihood approach that we believe is the best fit of the data. For simplicity purposes, we will only show time up to three years.

We define the table above as a complete data since we know specifically what each $n$ is. For incomplete data, we define $\left\{\left(r_{+k}, n_{+k}\right), k=1,2,3, \cdots, M\right\}$ and $n_{+k}=$ $\sum_{j=1}^{k} n_{j k}, k=1,2,3, \cdots, M$. In our case, we can write as $n_{+1}=n_{11}, n_{+2}=n_{12}+$ $n_{22}, n_{+3}=n_{13}+n_{23}+n_{33}$. In the incomplete data setting, we only know $n_{+k}$ but not the component within it.

Since we have two groups, AR and NR, we first define the likelihood function for each group. The likelihood functions for both groups have the similar form. 
Let

$$
\begin{aligned}
& z_{+j}=r_{+j}-n_{+j}, j=1,2,3 \\
& p_{+1}=\lambda_{11} \\
& p_{+2}=\lambda_{11}+\left(1-\lambda_{11}\right) \lambda_{12} \\
& p_{+3}=\lambda_{11}+\left(1-\lambda_{11}\right) \lambda_{12}+\left(1-\lambda_{11}\right)\left(1-\lambda_{12}\right) \lambda_{13} \\
& p_{+j}=\lambda_{11}+\sum_{k=1}^{j-1}\left[\prod_{i=1}^{k}\left(1-\lambda_{1 i}\right)\right] \lambda_{1(k+1)}, j=1,2,3, \ldots
\end{aligned}
$$

For incomplete data, let the likelihood functions of the first three time points be:

$$
\begin{aligned}
& L_{1}=p_{+1}^{n_{+1}}\left(1-p_{+1}\right)^{z_{+1}}=\lambda_{11}^{n_{+1}}\left(1-\lambda_{11}\right)^{r_{+1}-n_{+1}} \\
& \begin{aligned}
L_{2} & =p_{+2}^{n_{+2}}\left(1-p_{+2}\right)^{z_{+2}}=\left[\lambda_{11}+\left(1-\lambda_{11}\right) \lambda_{12}\right]^{n_{+2}}\left[\left(1-\lambda_{11}\right)\left(1-\lambda_{12}\right)\right]^{r_{+2}-n_{+2}} \\
L_{3} & =p_{+3}^{n_{+3}}\left(1-p_{+3}\right)^{z_{+3}} \\
& =\left[\lambda_{11}+\left(1-\lambda_{11}\right) \lambda_{12}+\left(1-\lambda_{11}\right)\left(1-\lambda_{12}\right) \lambda_{13}\right]^{n_{+3}}\left[\left(1-\lambda_{11}\right)\left(1-\lambda_{12}\right)\left(1-\lambda_{13}\right)\right]^{r_{+3}-n_{+3}} .
\end{aligned}
\end{aligned}
$$

The generalized form of the likelihood function at each time point can be written as:

$$
L_{j}=\left[\lambda_{11}+\sum_{k=1}^{j-1} \prod_{i=1}^{k}\left(1-\lambda_{1 i}\right) \lambda_{1(k+1)}\right]^{n_{+j}}\left[\prod_{k=1}^{j}\left(1-\lambda_{1 j}\right)\right]^{z_{+j}}, j=1,2, \ldots
$$


For complete data, assume the number of events follows a multinomial distribution. Let the likelihood functions of the first three time points be:

$$
\begin{aligned}
& L_{1}=\lambda_{11}^{n_{11}}\left(1-\lambda_{11}\right)^{z+1} \\
& L_{2}=\lambda_{11}^{n_{12}}\left[\left(1-\lambda_{11}\right) \lambda_{12}\right]^{n_{22}}\left[\left(1-\lambda_{11}\right)\left(1-\lambda_{12}\right)\right]^{z_{+2}} \\
& L_{3}=\lambda_{11}^{n_{13}}\left[\left(1-\lambda_{11}\right) \lambda_{12}\right]^{n_{23}}\left[\left(1-\lambda_{11}\right)\left(1-\lambda_{12}\right) \lambda_{13}\right]^{n_{33}}\left[\left(1-\lambda_{11}\right)\left(1-\lambda_{12}\right)\left(1-\lambda_{13}\right)\right]^{z_{+3}} .
\end{aligned}
$$

The generalized form of the likelihood function at each time point can be written as:

$$
L_{j}=\lambda_{11}^{n_{1 j}}\left[\left(1-\lambda_{11}\right) \lambda_{12}\right]^{n_{2 j}} \cdots\left[\prod_{k=1}^{j-1}\left(1-\lambda_{1 k}\right) \lambda_{1 j}\right]^{n_{j j}} \prod_{k=1}^{j}\left(1-\lambda_{1 k}\right)^{z_{+j}}
$$

We denote $\lambda_{1 k}$ the intensity rate for AR group, and $\lambda_{1 k}^{*}$ the intensity rate for NR group.

For AR group, the likelihood function is:

$$
\begin{aligned}
L_{A R}=\prod_{k=1}^{M} L_{k}= & \lambda_{11}^{\sum_{j=1}^{M} n_{1 j}} \lambda_{12}^{\sum_{j=2}^{M} n_{2 j}} \cdots \lambda_{1 k}^{\sum_{j=k}^{M} n_{k j}} \cdots \lambda_{1 M}^{n_{M M}} \\
& \times\left(1-\lambda_{11}\right)^{\left(\sum_{k=2}^{M} \sum_{j=k}^{M} n_{k j}+\sum_{j=1}^{M} z_{+j}\right)} \\
& \times\left(1-\lambda_{12}\right)^{\left(\sum_{k=3}^{M} \sum_{j=k}^{M} n_{k j}+\sum_{j=2}^{M} z_{+j}\right)} \\
& \times\left(1-\lambda_{13}\right)^{\left(\sum_{k=4}^{M} \sum_{j=k}^{M} n_{k j}+\sum_{j=3}^{M} z_{+j}\right)} \\
& \times \cdots\left(1-\lambda_{1, M-1}\right)^{n_{M M}+z_{M-1}+z_{M}}\left(1-\lambda_{1 M}\right)^{z_{+M}} \\
= & \prod_{k=1}^{M}\left\{\lambda_{1 k}^{\sum_{j=k}^{M} n_{k j}}\left(1-\lambda_{1 k}\right)^{\left(\sum_{i=k+1}^{M} \sum_{j=i}^{M} n_{i j}+\sum_{j=k}^{M} z_{+j}\right)}\right\}
\end{aligned}
$$


Similarly, for NR group the likelihood function is:

$$
L_{N R}=\prod_{k=1}^{M} L_{k}^{*}=\prod_{k=1}^{M}\left\{\lambda_{1 k}^{* \sum_{j=k}^{M} n_{k j}^{*}}\left(1-\lambda_{1 k}^{*}\right)^{\left(\sum_{i=k+1}^{M} \sum_{j=i}^{M} n_{i j}^{*}+\sum_{j=k}^{M} z_{+j}^{*}\right)}\right\}
$$

The log-likelihood function for the entire population would simply be the sum of $\log L_{A R}$ and $\log L_{N R}$.

$\lambda_{1 k}$ and $\lambda_{1 k}^{*}$ can be modeled as dependent using certain relationship so that both transition intensity rates can be included in one model. In addition, by establishing a relationship, we can replace one term with the other, which could potentially reduce the number of parameters in the calculation.

Assume there is a logit relationship between both group's transition intensity rates, defined as $e^{\beta}$, that is:

$$
\lambda_{1 k}^{*}=\frac{e^{\beta} \lambda_{1 k}}{1+\left(e^{\beta}-1\right) \lambda_{1 k}}, 1-\lambda_{1 k}^{*}=\frac{1-\lambda_{1 k}}{1+\left(e^{\beta}-1\right) \lambda_{1 k}}
$$

To derive the full complete log-likelihood function, we replace both $\lambda_{1 k}^{*}$ and $1-\lambda_{1 k}^{*}$ with terms above.

The full complete log-likelihood can be written as follow (denote $\theta=(\lambda, \beta)$ ): 


$$
\begin{aligned}
l_{c}(\lambda, \beta)=l_{c}(\theta)= & \sum_{k=1}^{M}\left\{\left(\sum_{j=k}^{M} n_{k j}\right) \log \lambda_{1 k}+\left(\sum_{i=k+1}^{M} \sum_{j=i}^{M} n_{i j}+\sum_{j=k}^{M} z_{+j}\right) \log \left(1-\lambda_{1 k}\right)\right\} \\
& +\sum_{k=1}^{M}\left[\sum_{j=k}^{M} n_{k j}^{*}\left\{\beta+\log \lambda_{1 k}-\log \left[1+\left(e^{\beta}-1\right) \lambda_{1 k}\right]\right\}\right. \\
& \left.+\left(\sum_{i=k+1}^{M} \sum_{j=i}^{M} n_{i j}^{*}+\sum_{j=k}^{M} z_{+j}^{*}\right)\left(\log \left(1-\lambda_{1 k}\right)-\log \left[1+\left(e^{\beta}-1\right) \lambda_{1 k}\right]\right)\right] \\
= & \sum_{k=1}^{M} \sum_{j=k}^{M}\left(n_{k j}+n_{k j}^{*}\right) \log \lambda_{1 k} \\
& +\sum_{k=1}^{M}\left(\sum_{i=k+1}^{M} \sum_{j=i}^{M}\left(n_{i j}+n_{i j}^{*}\right)+\sum_{j=k}^{M}\left(z_{+j}+z_{+j}^{*}\right)\right) \log \left(1-\lambda_{1 k}\right) \\
& -\sum_{k=1}^{M}\left(\sum_{j=k}^{M} n_{k j}^{*}+\sum_{i=k+1}^{M} \sum_{j=i}^{M} n_{i j}^{*}+\sum_{j=k}^{M} z_{+j}^{*}\right) \log \left(1+\left(e^{\beta}-1\right) \lambda_{1 k}\right) \\
& +n_{k j}^{*} \beta \\
& \sum_{j=k}(3.1) \\
& \\
& \\
&
\end{aligned}
$$

From the equation above we can obtain the sufficient statistics as follow:

$$
\Rightarrow \sum_{j=k}^{M}\left(n_{k j}+n_{k j}^{*}\right), \sum_{j=k}^{M} n_{k j}^{*}(k=1,2, \ldots, M)
$$

We assume $n_{+j}$ is known in the incomplete data and follows a binomial distribution. We can then estimate the distribution of $n_{k j}$. We calculate the condition distribution based on the incomplete likelihood function. 
That is, $n_{+j} \sim B\left(r_{+j}, 1-\prod_{i=1}^{j}\left(1-\lambda_{1 i}\right)\right), j=1,2, \ldots, M$

$$
\begin{gathered}
\Rightarrow n_{+j}=\sum_{k=1}^{j} n_{k j} \\
\Rightarrow n_{k j} \mid n_{+j} \sim B\left(n_{+j}, \frac{\prod_{i=1}^{k-1}\left(1-\lambda_{1 i}\right) \lambda_{1 k}}{1-\prod_{i=1}^{j}\left(1-\lambda_{1 i}\right)}\right), k=1,2, \ldots, j, j=1,2, \ldots, M .
\end{gathered}
$$

We can also obtain the expected value.

$$
\begin{aligned}
& E\left(n_{k j} \mid n_{+j}\right)=\frac{\lambda_{1 k} n_{+j} \prod_{i=1}^{k-1}\left(1-\lambda_{1 i}\right)}{\left[1-\prod_{i=1}^{j}\left(1-\lambda_{1 i}\right)\right]} \\
& E\left(n_{k j}^{*} \mid n_{+j}^{*}\right)=\frac{\lambda_{1 k}^{*} n_{+j}^{*} \prod_{i=1}^{k-1}\left(1-\lambda_{1 i}^{*}\right)}{\left[1-\prod_{i=1}^{j}\left(1-\lambda_{1 i}^{*}\right)\right]}
\end{aligned}
$$

For simplicity, let

$$
\begin{gathered}
t(k)=\sum_{j=k}^{M}\left(n_{k j}+n_{k j}^{*}\right) ; \\
t=(t(1), \ldots, t(M))^{T}
\end{gathered}
$$

We denote $N=\left\{n_{+k}, k=1,2, \ldots, M\right\}, N^{*}=\left\{n_{+k}^{*}, k=1,2, \ldots, M\right\}$.

Since

$$
\begin{gathered}
\left(n_{1 j}, n_{2 j}, \cdots, n_{j j}\right) \sim \operatorname{Multi}\left(r_{+j}, \lambda_{11},\left(1-\lambda_{11}\right) \lambda_{12},\left(1-\lambda_{11}\right)\left(1-\lambda_{12}\right) \lambda_{13}, \cdots, \prod_{i=1}^{j-1}\left(1-\lambda_{1 i}\right) \times \lambda_{1 j}\right) \\
\Rightarrow n_{k j} \sim B\left(r_{+j}, \prod_{i=1}^{k-1}\left(1-\lambda_{1 i}\right) \times \lambda_{1 k}\right)
\end{gathered}
$$


By applying EM Algorithm, we have the following two steps:

E-step:

$$
\begin{aligned}
G_{k}(\lambda, \beta) \triangleq E\left(t(k) \mid N, N^{*}\right)= & E\left(\sum_{j=k}^{M} n_{k j} \mid N\right)+E\left(\sum_{j=k}^{M} n_{k j}^{*} \mid N^{*}\right) \\
= & \sum_{j=k}^{M} E\left(n_{k j} \mid n_{+j}\right)+\sum_{j=k}^{M} E\left(n_{k j}^{*} \mid n_{+j}^{*}\right) \\
= & \sum_{j=k}^{M} \frac{n_{+j}}{1-\prod_{i=1}^{j}\left(1-\lambda_{1 i}\right)} \times \prod_{i=1}^{k-1}\left(1-\lambda_{1 i}\right) \times \lambda_{1 k} \\
& +\sum_{j=k}^{M} \frac{n_{+j}^{*}}{1-\prod_{i=1}^{j}\left(1-\lambda_{1 i}^{*}\right)} \times \prod_{i=1}^{k-1}\left(1-\lambda_{1 i}^{*}\right) \times \lambda_{1 k}^{*} \\
t^{(0)}= & \left(t^{(0)}(1), \cdots, t^{(0)}(M)\right)^{T}
\end{aligned}
$$

$t^{(0)}$ can be obtained by insert $\beta_{(0)}$ and $\lambda_{(0)}$ (initial values) into the function.

$$
t^{(0)}(k)=G_{k}(\lambda, \beta) \mid \lambda=\lambda_{(0)}, \beta=\beta_{(0)}
$$

M-step:

$$
\begin{aligned}
H_{k}(\lambda, \beta) \triangleq E(t(k)) & =\sum_{j=k}^{M}\left(E n_{k j}+E n_{k j}^{*}\right) \\
& =\prod_{i=1}^{k-1}\left(1-\lambda_{1 i}\right) \times \lambda_{1 k} \times \sum_{j=k}^{M} r_{+j}+\sum_{i=1}^{k-1}\left(1-\lambda_{1 i}^{*}\right) \lambda_{1 k}^{*} \sum_{j=k}^{M} r_{+j}^{*}
\end{aligned}
$$

Based on EM algorithm, we can obtain the values of $\lambda^{(m)}$ and $\beta^{(m)}$. From there we can then generate the values of $\lambda^{(m+1)}$ and $\beta^{((m+1)}$.

$$
\Rightarrow G_{k}\left(\lambda^{(m)}, \beta^{(m)}\right)=H_{k}\left(\lambda^{(m+1)}, \beta^{(m+1)}\right)
$$




$$
\begin{aligned}
\Rightarrow & \prod_{i=1}^{k-1}\left(1-\lambda_{1 i}^{(m+1)}\right) \times \lambda_{1 k}^{(m+1)} \sum_{j=k}^{M} r_{+j}+\sum_{i=1}^{k-1}\left(1-\lambda_{1 i}^{*(m+1)}\right) \lambda_{1 k}^{*(m+1)} \sum_{j=k}^{M} r_{+j}^{*} \\
= & \sum_{j=k}^{M} \frac{n_{+j}}{1-\prod_{i=1}^{j}\left(1-\lambda_{1 i}^{(m)}\right)} \prod_{i=1}^{k-1}\left(1-\lambda_{1 i}^{(m)}\right) \times \lambda_{1 k}^{(m)} \\
& \left.+\sum_{j=k}^{M} \frac{n_{+j}^{*}}{1-\prod_{i=1}^{j}\left(1-\lambda_{1 i}^{*(m)}\right)} \times \prod_{i=1}^{k-1}\left(1-\lambda_{1 i}^{*(m)}\right) \times \lambda_{1 k}^{*(m)}\right), k=1,2, \cdots, M(3.2)
\end{aligned}
$$

where

$$
\lambda_{1 k}^{*(m)}=\frac{e^{\beta^{(m)}} \lambda_{1 k}^{*(m)}}{1+\left(e^{\beta^{(m)}}-1\right) \lambda_{1 k}^{(m)}}
$$

$\lambda$ and $\beta$ are now obtained.

\subsection{Simulation Studies}

\subsubsection{Simulation Steps}

We define the true $\lambda$ and $\beta$ values and apply the method discussed in the last section to see how close the simulated values compared to the true values.

For simplification purpose, the simulation only considers three time points. The simulation is carrying out using the following steps:

1. Define initial values: $\lambda_{11}^{(0)}, \lambda_{12}^{(0)}, \lambda_{13}^{(0)}$ and $\beta$.

2. Compute $\lambda_{1 k}^{(1)}$ and $\beta^{(1)}$. In profile likelihood we assume $\beta$ is known.

i. Insert $\lambda_{1 k}^{(0)}$ into equation (3.1) to get $l_{c}\left(\lambda^{(0)}, \beta\right)=\lambda_{c}^{(0)}(\beta)$

By taking the derivative of the above equation with respect to beta and set to zero, we can solve for $\beta^{(1)}$. 
That is $\frac{\partial l_{c}^{(0)}(\beta)}{\partial \beta}=0 \Rightarrow \beta^{(1)}$

ii. Insert $\beta^{(0)}$ and $\lambda^{0)}$ into equation (3.2), we have

$$
G_{k}\left(\lambda^{(0)}, \beta^{(0)}\right)=H_{k}\left(\lambda^{(1)}, \beta^{(0)}\right) \Rightarrow \lambda_{1 k}^{(1)}, k=1,2,3 .
$$

3. $m^{\text {th }}$ iteration: compute $\lambda_{1}^{(m)}$ and $\beta^{(m)}$.

i. Insert $\lambda_{1 k}^{(m-1)}$ into equation (3.1) to get $l_{c}\left(\lambda_{1}^{(m-1)}, \beta\right)=\lambda_{c}^{(m-1)}(\beta)$

By taking the derivative of the above equation with respect to beta and set to zero, we can solve for $\beta^{(m)}$.

That is $\frac{\partial l_{c}^{(m-1)}(\beta)}{\partial \beta}=0 \Rightarrow \beta^{(m)}$

ii. Insert $\lambda_{1 k}^{(m-1)}$ and $\beta^{(m-1)}$ into equation (3.2), we have

$$
G_{k}\left(\lambda^{(m-1)}, \beta^{(m-1)}\right)=H_{k}\left(\lambda^{(m)}, \beta^{(m-1)}\right) \Rightarrow \lambda_{1 k}^{(m)}, k=1,2,3 .
$$

4. If the distance between $\left(\lambda_{11}^{(m)}, \lambda_{12}^{(m)}, \lambda_{13}^{(m)}, \beta^{(m)}\right)$ and $\left(\lambda_{11}^{(m-1)}, \lambda_{12}^{(m-1)}, \lambda_{13}^{(m-1)}, \beta^{(m-1)}\right)$ is smaller than a pre-selected $C_{0}$, we stop the iteration and report $\hat{\lambda_{1 k}}=\lambda_{1 k}^{(m)},(k=$ $1,2,3)$ and $\hat{\beta}=\beta^{(m)}$.

We can then use these two values to get $\lambda_{1 k}^{*}$.

$$
\Rightarrow \lambda_{1 k}^{*}=\frac{\hat{\lambda_{1 k}} e^{\hat{\beta}}}{1+\left(e^{\hat{\beta}}-1\right) \hat{\lambda_{1 k}}}, k=1,2,3 .
$$

\subsubsection{Simulation Results}

We calculated $\lambda$ and $\beta$ with different sample size assumptions. In reality, we assume the number of subjects is always larger in the AR group compared to the NR group. 
We also tested for equal sample size just for demonstration purposes.

Simulated results can be found in Table 3.1. The values of simulated $\beta$ are very close to the true $\beta$ value which suggests that the relationship between two groups' transition intensity rates have maintained through the simulation. Regardless the size or the ratio of the sample sizes, all simulated values tend to converge to the true value. Visualized results can be found in Figure 3.1 (for AR group) and Figure 3.2 (for NR group).

We also report the confidence intervals, IQR, as well as standard deviation (Table 3.2). The confidence intervals are calculated based on mean and standard error while the IQR are calculated from $25 \%$ and $75 \%$ quantile.

\subsection{Discussion}

In this project, we applied the EM algorithm with the profile likelihood for estimating transition intensity rate under the semi-parametric setting. We employed a logit relationship between transition intensity rates from different risk groups. The data-driven likelihood function includes almost all the characteristics of the dataset. Though for demonstration purposes we only considered a few time points, one could easily apply

the approach to a much larger dataset. By employing proposed approach, one could also save time during calculation due to the reduction of parameters.

When the dataset has a small sample size, one could combine time points together and estimate the transition intensity rates. If the sample size is large, yearly rates should be considered as described in this project.

The relationship between $\lambda$ and $\lambda^{*}$ is extremely important. Here we assume a logit relationship based on Cox's proportional model (Cox, 1972), but other approaches can also be considered. For example, one could consider using $\lambda=\lambda^{*}+\gamma$ which is called an additive failure rate approach in Rai and Matthews (1997). We did not consider the rate dependent on the exponential distribution as Rai et al. (2013) 
did. However, one could easily extend the methodology to include piecewise exponential.

For simplification purposes the methodology is built upon a special case twostate model. Future research will extend the methodology to a complete three-state model, which in that case, more parameters will be included. 


\subsection{Tables and Figures}

Figure 3.1: Corresponding $\lambda$ values with different sample size assumptions (AR group).

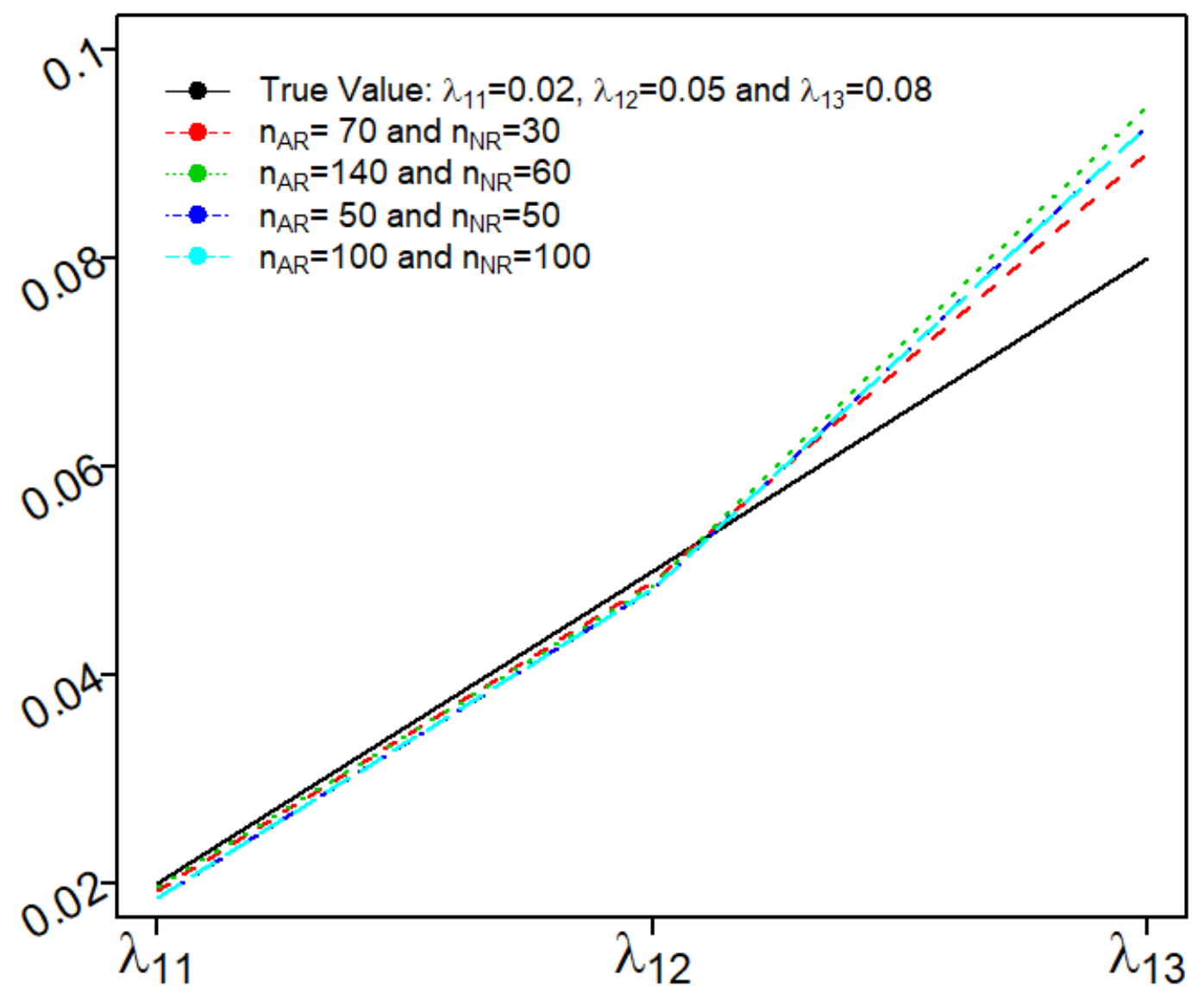


Figure 3.2: Corresponding $\lambda$ values with different sample size assumptions (NR group).

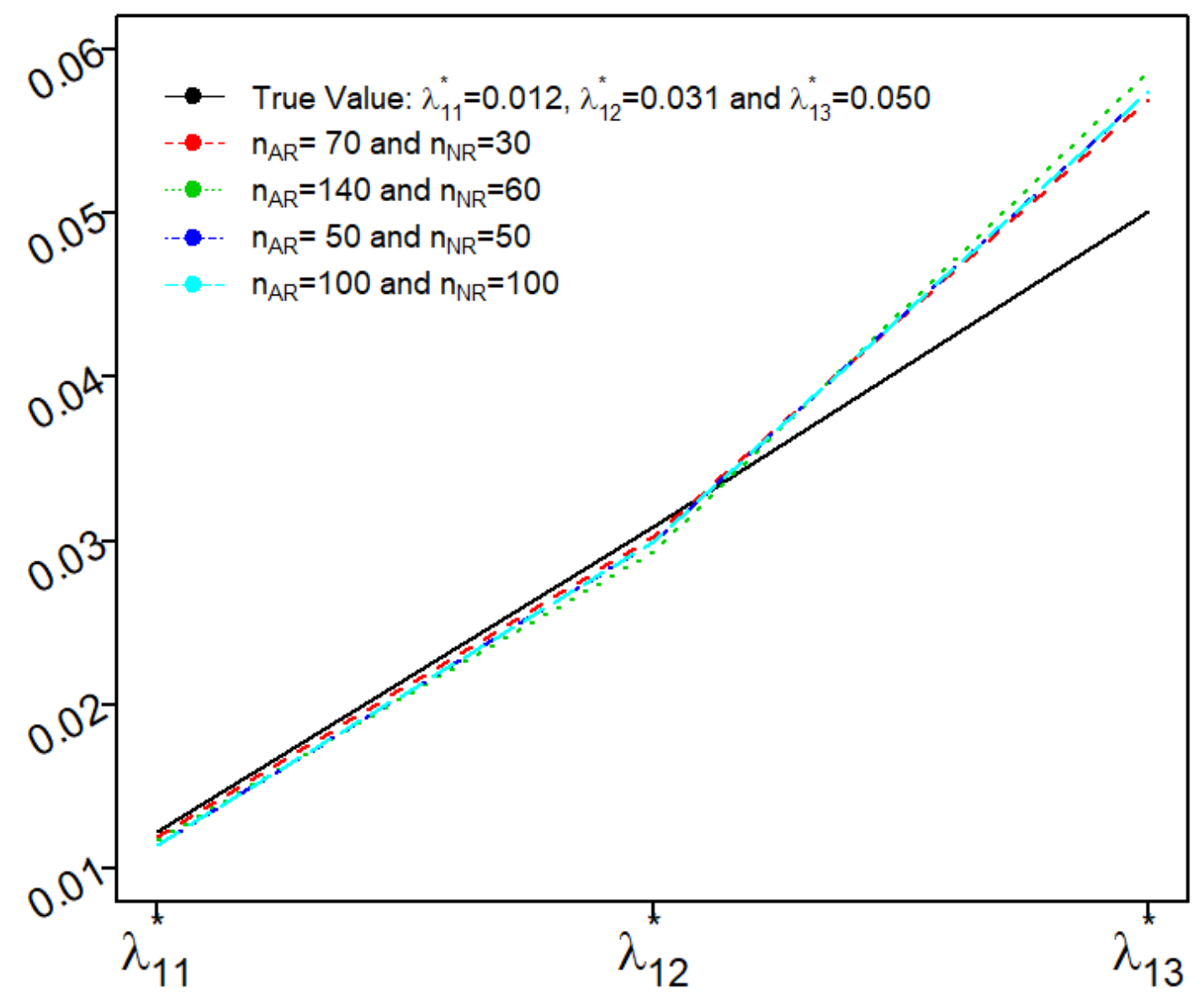


Table 3.1: Comparison of simulated mean $\lambda$ and $\beta$ values with the true value.

\begin{tabular}{|c|c|c|c|c|c|c|c|c|c|}
\hline & $n_{A R}$ & $n_{N R}$ & $\beta$ & $\lambda_{11}$ & $\lambda_{12}$ & $\lambda_{13}$ & $\lambda_{11}^{*}$ & $\lambda_{12}^{*}$ & $\lambda_{13}^{*}$ \\
\hline \multirow{2}{*}{ True Value } & & & -0.5000 & 0.0200 & 0.0500 & 0.0800 & 0.0122 & 0.0309 & 0.0501 \\
\hline \multirow{3}{*}{ Mean } & 50 & 50 & -0.5477 & 0.0187 & 0.0482 & 0.0928 & 0.0114 & 0.0300 & 0.0574 \\
\cline { 2 - 9 } & 70 & 30 & -0.5873 & 0.0193 & 0.0489 & 0.0901 & 0.0119 & 0.0303 & 0.0569 \\
\cline { 2 - 9 } & 100 & 100 & -0.5520 & 0.0192 & 0.0500 & 0.0919 & 0.0113 & 0.0297 & 0.0560 \\
\cline { 2 - 9 } & 140 & 60 & -0.5660 & 0.0196 & 0.0486 & 0.0946 & 0.0117 & 0.0293 & 0.0587 \\
\hline
\end{tabular}


Table 3.2: Confidence intervals, interquartile range, and standard deviations for $\lambda$ and $\beta$ with different sample size.

\begin{tabular}{|c|c|c|c|c|c|c|}
\hline$n_{A R}$ & $n_{N R}$ & & Mean & Confidence Interval & IQR & Standard Deviation \\
\hline \multirow{7}{*}{70} & \multirow{7}{*}{30} & $\beta$ & -0.5873 & $(-0.6240,-0.5506)$ & $(-0.9024,-0.2004)$ & 0.5917 \\
\hline & & $\lambda_{11}$ & 0.0193 & $(0.0185,0.0202)$ & $(0.0105,0.0288)$ & 0.0136 \\
\hline & & $\lambda_{12}$ & 0.0489 & $(0.0473,0.0506)$ & $(0.0305,0.0673)$ & 0.0268 \\
\hline & & $\lambda_{13}$ & 0.0901 & $(0.0872,0.0929)$ & $(0.0579,0.1208)$ & 0.0456 \\
\hline & & $\lambda_{11}^{*}$ & 0.0119 & $(0.0113,0.0125)$ & $(0.0051,0.0170)$ & 0.0101 \\
\hline & & $\lambda_{12}^{*}$ & 0.0303 & $(0.0290,0.0316)$ & $(0.0134,0.0438)$ & 0.0212 \\
\hline & & $\lambda_{13}^{*}$ & 0.0569 & $(0.0545,0.0592)$ & $(0.0285,0.0794)$ & 0.0375 \\
\hline \multirow{7}{*}{140} & \multirow{7}{*}{60} & $\beta$ & -0.5660 & $(-0.5916,-0.5404)$ & $(-0.8343,-0.2855)$ & 0.4132 \\
\hline & & $\lambda_{11}$ & 0.0196 & $(0.0189,0.0202)$ & $(0.0118,0.0254)$ & 0.0101 \\
\hline & & $\lambda_{12}$ & 0.0486 & $(0.0474,0.0498)$ & $(0.0348,0.0612)$ & 0.0199 \\
\hline & & $\lambda_{13}$ & 0.0946 & $(0.0925,0.0967)$ & $(0.0728,0.1159)$ & 0.0332 \\
\hline & & $\lambda_{11}^{*}$ & 0.0117 & $(0.0112,0.0121)$ & $(0.0067,0.0156)$ & 0.0070 \\
\hline & & $\lambda_{12}^{*}$ & 0.0293 & $(0.0284,0.0302)$ & $(0.0191,0.0376)$ & 0.0148 \\
\hline & & $\lambda_{13}^{*}$ & 0.0587 & $(0.0570,0.0605)$ & $(0.0387,0.0758)$ & 0.0278 \\
\hline \multirow{7}{*}{50} & \multirow{7}{*}{50} & $\beta$ & -0.5477 & $(-0.5822,-0.5131)$ & $(-0.8760,-0.1761)$ & 0.5574 \\
\hline & & $\lambda_{11}$ & 0.0187 & $(0.0178,0.0196)$ & $(0.0100,0.0277)$ & 0.0145 \\
\hline & & $\lambda_{12}$ & 0.0482 & $(0.0464,0.0500)$ & $(0.0268,0.0675)$ & 0.0291 \\
\hline & & $\lambda_{13}$ & 0.0928 & $(0.0897,0.0960)$ & $(0.0562,0.1268)$ & 0.0511 \\
\hline & & $\lambda_{11}^{*}$ & 0.0114 & $(0.0108,0.0120)$ & $(0.0049,0.0169)$ & 0.0097 \\
\hline & & $\lambda_{12}^{*}$ & 0.0300 & $(0.0287,0.0313)$ & $(0.0155,0.0421)$ & 0.0205 \\
\hline & & $\lambda_{13}^{*}$ & 0.0574 & $(0.0552,0.0595)$ & $(0.0307,0.0786)$ & 0.0348 \\
\hline \multirow{8}{*}{100} & \multirow{8}{*}{100} & & & & & \\
\hline & & $\beta$ & -0.5520 & $(-0.5731,-0.5310)$ & $(-0.7652,-0.3168)$ & 0.3396 \\
\hline & & $\lambda_{11}$ & 0.0192 & $(0.0185,0.0199)$ & $(0.0120,0.0260)$ & 0.0108 \\
\hline & & $\lambda_{12}$ & 0.0500 & $(0.0487,0.0514)$ & $(0.0353,0.0642)$ & 0.0220 \\
\hline & & $\lambda_{13}$ & 0.0919 & $(0.0897,0.0942)$ & $(0.0671,0.1155)$ & 0.0364 \\
\hline & & $\lambda_{11}^{*}$ & 0.0113 & $(0.0109,0.0117)$ & $(0.0067,0.0152)$ & 0.0067 \\
\hline & & $\lambda_{12}^{*}$ & 0.0297 & $(0.0288,0.0305)$ & $(0.0197,0.0380)$ & 0.0138 \\
\hline & & $\lambda_{13}^{*}$ & 0.0560 & $(0.0545,0.0575)$ & $(0.0386,0.0717)$ & 0.0244 \\
\hline
\end{tabular}




\section{CHAPTER 4 \\ ESTIMATING CUMULATIVE INCIDENCE RATE ON INTERVAL CENSORED DATA IN AN ILLNESS-DEATH MODEL}

\subsection{Introduction}

\subsubsection{Background}

In the first project, we discussed about the issue of missing information on interval censored data. We proposed an imputation approach to overcome the issue, and through simulation, we concluded that the imputed data was much efficient than the one without. In the second project, we proposed a new approach to estimate the transition intensity probabilities on interval censored data in an illness/death model. We established a logit relationship between transition intensity rates, obtained from each risk group respectfully. We later applied the EM algorithm with profile likelihood to obtain the final outcome. Simulation studies also prove that the approach is valid since the true value and simulated values were very close.

However, proposed approaches in both projects are built upon a special case illness/death model which no death has occurred. In that case, we only have event cases (patients with abnormality) and censored cases. The special case two-state model provides us the convenience to validate our methodologies with the option to easily extend to a complete three-state model.

Regardless of the model, our primary interest is still $\lambda_{1}$, the transition intensity rate for patients progressing towards cardiac abnormal. However, the situation 
is getting a little bit more complicated when some patients progress from the initial state directly to the absorbing state, which is $\lambda_{3}$ on Figure 1.1. This is known as the competing risk events of $\lambda_{1}$.

In this project, we will be estimating $\lambda_{1}$ incorporating information from both $\lambda_{2}$ and $\lambda_{3}$. With additional information included, the result is expected to be more accurate. We will be carrying out both parametric approach, as described in Rai et al. (2013), and semi-parametric approach, as described in the second project. However, in this dissertation we will only show the parametric approach. The semi-parametric approach can be later found in a published article.

\subsubsection{Methodology Review}

In longitudinal failure time data, multi-state models are among the mostly used models when examining the event outcomes. Commegenes (2002) provided an overview of the inference for nonparametric approaches for the multi-state model. Ruiz-Castro and Zenga (2020) discussed the use of a general piecewise multi-state survival model. The illness-death model is one of the simple cases of the multi-state model. $R$ packages such as TP.idm can be used to estimate the transition probabilities in an illness-death model through a nonparametric approach (Balboa and Una-Alvarez, 2018).

Competing risk does not become a neglected topic in survival analysis. When competing events occur, basic Kaplan-Meier estimation procedure is not usually applicable as it is unable to account for the information passed from the competing

events. In time-to-event data, Stagopan et al. (2004) introduced a nonparametric approach incorporating competing risk and compared with Kaplan-Meier estimation approach and found that failing to account for competing events might lead to an overestimation of the cumulative incidence.

Under the interval censored competing risk data, Kim and Kim (2016) dis- 
cussed the analysis by using a pseudo-value approach and found it performed well under various scenarios. Mao et al. (2017) introduced a semi-parametric approach for handling such dataset. Mostly recently, Park et al. (2021) developed a comprehensive analysis of interval censored data with competing events and missing events. $R$ package such as intccr has been developed and can be easily used to handle such data (Park et al., 2019). In the illness-death model, Hinchliffe et al. (2013) presented the theory of using a flexible parametric survival model approach. More information regarding mutli-state models for interval censored data can be found in van den Hout (2020).

\subsection{Method}

The parametric method used in this project is an extension from Rai et al. (2013). Detailed approach of getting the likelihood function can be found in Appendix 1. After getting the full likelihood function, we calculate the score function and the information matrix.

Let $\lambda$ be the vector of $\lambda_{1}, \lambda_{2}, \lambda_{3}$.

The score function can be defined as:

$$
S(\lambda)=\left(\frac{\partial l}{\partial \lambda_{1}}, \frac{\partial l}{\partial \lambda_{2}}, \frac{\partial l}{\partial \lambda_{3}}\right)^{T}
$$

The information matrix can be defined as:

$$
\sum(\lambda)=\left(\frac{\partial^{2} l}{\partial \lambda_{i} \partial \lambda_{j}}\right)_{3 \times 3}
$$


Detailed derivations and final form of their components can be found in Appendix 2. Through Newton-Raphson method, we can obtain $\lambda$ using the following formula:

$$
\Rightarrow \lambda^{(p+1)}=\lambda^{(p)}-s^{(p)} \sum\left(\lambda^{(p)}\right) S\left(\lambda^{(p)}\right),\left(0<s^{(p)} \leq 1\right)
$$

where $s^{(p)}$ is a coefficient between 0 and 1 . The purpose of this term, known as the restraint coefficient for the Newton-Raphson method, is to make sure the second part of the function does not exceed 1 .

The semi-parametric method is a modified updated version of the one that was introduced in the second project. Detailed approach and application will be available in a future published manuscript.

\subsection{Data Application}

\subsubsection{Data Information}

In the previous two projects, the group classification (AR or NR) was defined based on variables $\mathrm{AF}$ and $\mathrm{FS}$. In the updated dataset, in addition to these two variables, we include two new measurements: systemic vascular resistance (SVR) and ejection fraction (EF). Based on experienced physicians' point of view, SVR and EF are more suitable for determining the cardiac condition of patients and both measurements are more often used in the real clinical setting.

SVR is the calculated amount of force for left ventricular afterload. It represents the resistance in the circulatory system that is used to pump and circulate blood (Abbas et al., 2004; Almodovar et al., 2011). According to the American Society of Echocardiography's guidelines and standards, SVR can be calculated by using the 
following formula:

$$
S V R\left(\text { dynes } \times \mathrm{sec} / \mathrm{cm}^{5}\right)=\frac{M A P-R A P(\mathrm{~mm} \mathrm{Hg})}{C O(L / \text { min })} \times 80
$$

where $M A P$ represents the mean arterial pressure, $R A P$ represents the mean right atrial pressure, and $C O$ represents the cardiac output (Porter et al., 2015). The normal range for SVR is between 900 and 1440.

$\mathrm{EF}$ is a measurement of the percentage of blood pumped out from the left ventricle at each contraction (Mayo Clinic, accessed on March 16, 2021). According to the American Heart Association, the normal range of $\mathrm{EF}$ is between $50 \%$ and $75 \%$.

The distribution of the dataset can be found in the table below.

\begin{tabular}{|l|c|c|c|c|c|}
\hline \multirow{2}{*}{} & \multicolumn{2}{|c|}{ Cardiac Normal } & \multicolumn{2}{c|}{ Cardiac Abnormal } & \\
\cline { 2 - 6 } & Alive & Death & Alive & Death & Total \\
\hline At-Risk & 1345 & 42 & 180 & 24 & 1591 \\
\hline Not-At-Risk & 252 & 1 & 10 & 1 & 264 \\
\hline Total & 1597 & 43 & 190 & 25 & 1855 \\
\hline
\end{tabular}

Notice that in the NR group, death cases in both normal and abnormal patients are relatively small.

\subsubsection{Application Result}

Note that the primary purpose of this data application is to demonstrate statistical methods and to show the importance of estimating cumulative incidence rates, readers should not draw clinical conclusions from this project. 
We obtained all three transition intensity rates $\left(\lambda_{1}, \lambda_{2}\right.$ and $\left.\lambda_{3}\right)$ though our primary interest is $\lambda_{1}$. The transition intensity rate for the AR group is 0.0143 . Similarly, the NR group has the transition intensity rate of 0.0096 (Table 4.1). Group effect appears to be significantly different with p-value less than 0.001 . The rate for $\mathrm{AR}$ is greater than NR proves the assumption that the incidence rate is much higher for patients treated with cardiac radiation or anthracycline.

As noted before, the transition intensity rate alone cannot produce valuable information to the researchers. Reporting the cumulative incidence rate is more ideal. Here we report the fixed-term cumulative incidence rate based on $\lambda_{1}$ (Table 4.2). By five years, the difference in cumulative incidence rates tend to be small ( $2 \%$ difference). This suggests that at the beginning of the followup, intervention might not be needed since AR and NR groups have similar cumulative incidence rates. However, when the patients reach Year 10, the difference is a little bit over 5\%, and by Year 20, the difference is approximately 10\%. This suggests that somewhere in between, an intervention should be considered. The visualized plot can be found in Figure 4.1.

\subsection{Discussion}

In this project we applied parametric and semi-parametric methods on a new dataset in a three-state illness/death model. The new dataset actually has more than 4000 observations, but we only managed to use less than half of it. The issue is similar to the one that was described in the first project. To declare whether or not a patient is cardiac abnormal, several outcome variables can be used. For ejection fraction, it is just one single variable, but for SVR, it is calculated from three different measurements. If one of these measurements is not available for any unspecified reason, then we are not able to calculate SVR, and therefore, we could not determine the patient's heart condition. Even if the patient has a normal AF value, we cannot assume that the SVR is normal as well. Correlation tests need to be carried out to see if these 
variables are in fact related. Afterwards, proper imputation, as discussed in the first project, should be taken to avoid the loss of valuable information.

Similar issues might also impact the distribution of the data as well as the outcome. In our dataset, the ratio of two risk groups tends to be large. This can be true because most cancer patients were treated with cardiac radiation or anthracycline, but it also might be caused by the missing observations. In the NR group, death cases are very few. This is either because the NR group patients are more likely to live longer, or because there are missing observations we did not include in the analysis. Valuable information is in fact lost. There are several literature discussions about the analysis on interval censored competing risk data with missing information. Lee and Kim (2018) and Li, Luderer, and Baker (2014) proposed using imputation to handle such issues. Mitra, Das and Das (2019) proposed an approach that utilizes available data to analyze the interval censored competing risk data.

In the parametric setting, we assume the rate follows an exponential distribution which means the rate is constant at all time points. Though it is much easier to calculate because the number of parameters is much smaller, this assumption might not always be true. As a result, we suggest using a semi-parametric approach to obtain a more accurate outcome. 


\subsection{Tables and Figures}

Figure 4.1: Fixed-term cumulative incidence rates for AR and NR groups $\left(\lambda_{1}\right)$.

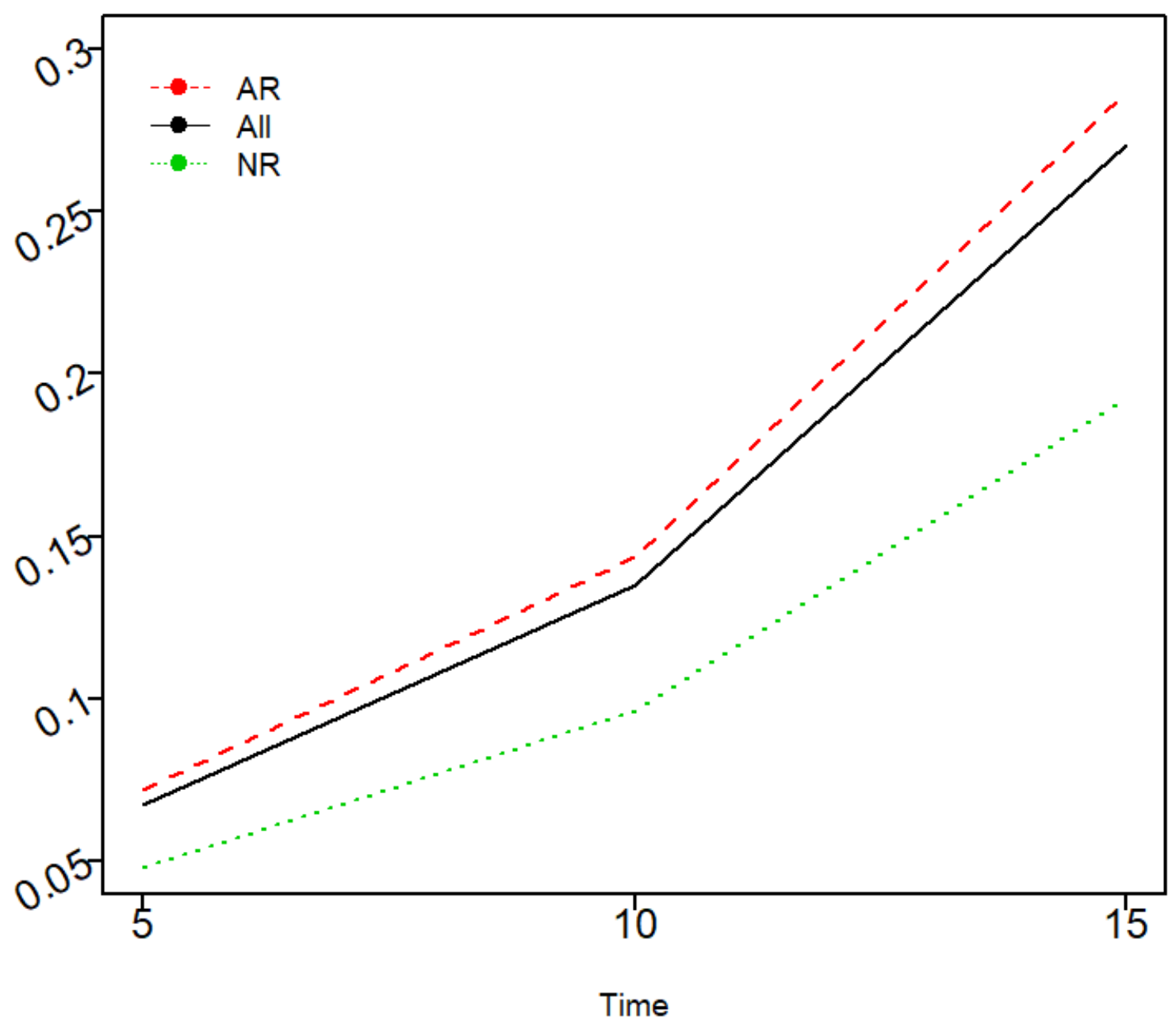


Table 4.1: Transition intensity rates for AR and NR groups.

\begin{tabular}{|c|c|c|c|}
\hline Incidence Rate & $\lambda_{1}$ & $\lambda_{2}$ & $\lambda_{3}$ \\
\hline All (n=1855) & 0.0135 & 0.0036 & 0.0104 \\
\hline AR (n=1591) & 0.0143 & 0.0038 & 0.0104 \\
\hline NR (n=264) & 0.0096 & 0.0011 & 0.0107 \\
\hline
\end{tabular}


Table 4.2: Fixed-term cumulative incidence rates for AR and NR groups.

\begin{tabular}{|c|c|c|c|}
\hline 5-Year CIF & $\lambda_{1}$ & $\lambda_{2}$ & $\lambda_{3}$ \\
\hline All (n=1855) & 0.0675 & 0.0173 & 0.0522 \\
\hline AR (n=1591) & 0.0717 & 0.0192 & 0.0522 \\
\hline NR (n=264) & 0.0481 & 0.0056 & 0.0537 \\
\hline \multicolumn{5}{|l}{} \\
\hline 10-Year CIF & $\lambda_{1}$ & $\lambda_{2}$ & $\lambda_{3}$ \\
\hline All (n=1855) & 0.1351 & 0.0346 & 0.1045 \\
\hline AR (n=1591) & 0.1435 & 0.0384 & 0.1044 \\
\hline NR (n=264) & 0.0962 & 0.0112 & 0.1074 \\
\hline \multicolumn{5}{|l|}{} & $\lambda_{2}$ & $\lambda_{3}$ \\
\hline 20-Year CIF & $\lambda_{1}$ & 0.0691 & 0.2089 \\
\hline All (n=1855) & 0.2703 & 0.0768 & 0.2087 \\
\hline AR (n=1591) & 0.2869 & 0.0225 & 0.2149 \\
\hline NR (n=264) & 0.1924 &
\end{tabular}




\section{FUTURE WORK}

We have established the fundamental theory of analyzing interval censored data in an illness/death model, specifically for childhood cancer survivors treated with chest radiation or anthracycline. With additional information (variables) on hand, we can incorporate each of them into the model to help improve the accuracy of the outcome. We believe that, by estimating the cumulative incidence rates, physicians could gain insightful knowledge of their patients as well as the treatments they received. In future works, we will incorporate comorbidity information into the model. We will also use measurements from multi-visits to create a longitudinal model. Last but not the least, we will develop a modified model to incorporate gene expression data. With better estimation, cardiotoxicity can be prevented and lives can be saved. 


\section{REFERENCES}

[1] Abbas, A.E., Fortuin, F.D., Patel, B., Moreno, C.A., and Schiller, N.B. (2004). Noninvasive measurement of systemic vascular resistance using Doppler echocardiography. Journal of the American Society of Echocardiography, 17(8):834-838.

[2] American Cancer Society. (2020). Cancer Facts 86 Figures 2020. American Cancer Society. Atlanta, GA.

[3] American Cancer Society. (2021). Cancer Facts \& Figures 2021. American Cancer Society. Atlanta, GA.

[4] Armstrong G.T., Chen, Y., Yasui, Y., Leisenring, W., Gibson, T.M., Mertens, A.C., et al. (2016). Reduction in late mortality among 5-year survivors of childhood cancer. The New England Journal of Medicine, 374(9):833-42.

[5] Armstrong, G.T., Liu, Q., Yasui, Y., Neglia, J.P., Leisenring, W.L., Robison, L.L., et al. (2009). Late mortality among 5-year survivors of childhood cancer: a summary from the childhood cancer survivor study. Journal of Clinical Oncology, 27(14):2328-2338.

[6] Ayer, M., Brunk, H.D., Ewing, G.M., Reid W.T., and Silverman E. (1955). An empirical distribution function for sampling with incomplete information. The Annals of Mathematical Statistics, 26:641-647.

[7] Balboa, V. and Una-Alvarez, J.D. (2018). Estimation of transition probabilities for the illness-death model: Package TP.idm. Journal of Statistical Software, 83:10. 
[8] Chow, S.C. and Liu, J.P. (2013). Design and analysis of clinical trials: concepts and methodologies. John Wiley Sons, Inc, Hoboken, NJ, 2nd edition.

[9] Cohen, J. (1988). Statistical power analysis for the behavioral sciences. Lawrence Erlbaum Associates, Hillsdale, NJ, 2nd edition.

[10] Colan, S.D., Parness, I.A., Spevak, P.J., and Sanders, S.P. (1992). Developmental modulation of myocardial mechanics: Age- and growth-related alterations in afterload and contractility. Journal of the American College of Cardiology, 19:619629.

[11] Commenges, D. (2002). Inference for multi-state models from interval-censored data. Statistical Methods in Medical Research, 11(2):167-182.

[12] Cox, D. R. (1972). Regression Models and Life-Tables. Journal of the Royal Statistical Society: Series B (Methodological), 34(2):187-220.

[13] Dodge Y. (2003). The Oxford Dictionary of Statistical Terms. The International Statistical Institute, Oxford Press.

[14] Fidler, M.M., Reulen, R.C., Winter, D.L., et al. (2016). Long term cause specific mortality among 34489 five year survivors of childhood cancer in Great Britain: population based cohort study. British Medical Journal, 354: i4351.

[15] Goetghebeur, E. and Ryan, L. (2000). Semiparametric regression analysis of interval-censored data. Biometrics, 56(4):1139-1144.

[16] Gunaratnam, B. and Rai, S.N. (2019). Comparing the variability using Louis' method and resampling methods. Journal of Biometrics and Biostatistics, 10(1).

[17] Henry, W.L., Gardin, J.M., and Ware, J.H. (1980). Echocardiographic measurements in normal subjects from infancy to old age. Circulation, 62:1054-1061. 
[18] Hinchliffe, S.R., Scott, D.A., and Lambert, P.C. (2013). Flexible parametric illness-death models. The Stata Journal, 13(4):759-775.

[19] Howlader, N., Noone, A.M., Krapcho, M., et al. (2015). SEER Cancer Statistics Review (CSR), 1975-2012. Bethesda,MD: National Cancer Institute (internet). http://https://seer.cancer.gov/archive/csr/1975_2012/ (accessed November $18,2020)$.

[20] Huang, J. (1995). Maximum likelihood estimation for proportional odds regression model with current status data. Lecture Notes-Monograph Series, 27:129-145.

[21] Huang, J. (1996). Efficient estimation for the proportional hazards model with interval censoring. The Annals of Statistics, 24(2):540-568.

[22] Hudson, M.M., Ness, K.N., Nolan, V.G., Armstong, G.T., Green, D.M., Moris, E.B., et al. (2011). Prospective medical assessment of adults surviving childhood cancer: study design, cohort characteristics, and feasibility of the St. Jude Lifetime Cohort study. Pediatric Blood Cancer, 56(5):825-836.

[23] Hudson, M.M., Rai, S.N., Nunez, C., Merchant, T.E., Marina, N.M., Zalamea, N., et al. (2007). Noninvasive evaluation of late anthracycline cardiac toxicity in childhood cancer survivors. Journal of Clinical Oncology, 25:3635-3643.

[24] Huque, M.H., Carlin, J.B., Simpson, J.A., and Lee, K.J. (2018). A comparison of multiple imputation methods for missing data in longitudinal studies. BMC Medical Research Methodology, 18:168.

[25] Kaplan, E.L. and Meier, P. (1958). Nonparametric estimation from incomplete observation. Journal of the American Statistical Association, 53:457-481. 
[26] Kenward, M.G. and Carpenter, J.R. (2009). Multiple Imputation. In Handbooks of Modern Statistical Methods; Longitudinal Data Analysis, Chapman Hall/CRC, Florida, Chapter 21, pages 477-500.

[27] Kim, S. and Kim, Y. (2016). Regression analysis of interval censored competing risk data using a pseudo-value approach. Communications for Statistical Applications and Methods, 23:555-562.

[28] King, G., Honaker, J., Joseph, A., and Scheve, K. (2001). Analyzing incomplete political science data: an alternative algorithm for multiple imputation. The American Political Science Review, 95:49-69.

[29] Koutsoukis, A., Ntalianis, A., Repasos, E., Kastritis, E., Dimopoulos, M.A., and Paraskevaidis, I. (2018). Cardio-oncology: A Focus on Cardiotoxicity. European Cardiology Review, 13(1):64-69.

[30] Krischer, J.P., Epstein, S., Cuthbertson, D.D., Goorin, A.M., Epstein, M.L., and Lipshultz, S.E. (1997). Clinical cardiotoxicity following an-thracycline treatment for childhood cancer: The Pediatric Oncology Group experience. Journal of Clinical Oncology, 15:1544-1552.

[31] Lee, H.E. and Kim, Y.J. (2020). Analysis of interval censored competing risk data via nonparametric multiple imputation. Statistics in Biopharmaceutical Research, DOI: $10.1080 / 19466315.2020 .1741445$

[32] Li, T., Luderer, U., and Baker, D. (2014). Exploring the method for analyzing interval censored data using imputation in competing risks mode. Joint Statistical Meeting, Survey Research Methods Section. http://www.asasrms.org/Proceedings/y2014/files/311780_88136.pdf 
[33] Lipshultz, S., Cochran, T., Franco, V., and Miller, T.L. (2013). Treatmentrelated cardiotoxicity in survivors of childhood cancer. Nature Review Clinical Oncology, 10:697-710.

[34] Little, R.J. and Rubin, D.B. (2002). Statistical Analysis with Missing Data, John Wiley, New York, 2nd edition.

[35] Liu, C. (1995). Missing data imputation using the multivariate t distribution. Journal of Multivariate Analysis, 53:139-158.

[36] Liu, Y. and De, A. (2015). Multiple imputations by fully conditional specification for dealing with missing data in a large epidemiologic study. International Journal of Statistics in Medical Research, 4(3):287-295.

[37] Mayo Clinic. Ejection faction: what does it measure? (Internet) https://www.mayoclinic.org/ejection-fraction/expert-answers/faq-20058286 (accessed March 16, 2021).

[38] Mao, L., Lin, D.Y., and Zeng, D. (2017). Semiparametric regression analysis of interval-censored competing risks data. Biometrics, 73(3):857-865.

[39] Almodovar, M.C., Kulik, T.J., and Charpie, J.R. (2011). Assessment of cardiovascular function in Pediatric Critical Care, Mosby, 4th edition.

[40] Mitra, D., Das, U., and Das, K. (2020). Analysis of interval-censored competing risks data under missing causes. Journal of Applied Statistics, 47:3,439-459.

[41] Molenberghs, G. and Fitzmoaurice, G. (2009). Incomplete data: Introduction and overview. In Handbooks of Modern Statistical Methods; Longitudinal Data Analysis, Chapman Hall/CRC, Florida, Chapter 17, pages 395-408.

[42] Murphy, S.A. and Van der Vaart, A.W. (2000). On Profile Likelihood. Journal of the American Statistical Association, 95:450,449-465. 
[43] Park, J., Bakoyannis, G., and Yiannoutsos, C.T. (2019). Semiparametric competing risks regression under interval censoring using the $\mathrm{R}$ package intccr. Computer Methods and Programs in Biomedicine, 173:167-176.

[44] Park, J., Bakoyannis, G., Zhang, Y., and Yiannoutsos, C.T. (2021). Semiparametric regression on cumulative incidence function with interval-censored competing risks data and missing event types. Biostatistics, kxaa052.

[45] Pein, F., Sakiroglu, O., Dahan, M., Lebidois, J., Merlet, P., Shamsaldin., et al. (2004). Cardiac abnormalities 15 years and more after adriamycin therapy in 229 childhood survivors of a solid tumor at the Institut Gustave Roussy. British Journal of Cancer, 91:37-44.

[46] Peto, R. (1973). Experimental survival curves for interval-censored data. Applied Statistisc, 22:86-91.

[47] Phillips, S.M., Padgett, L.S., Leisenring, W.M., Stratton, K.K., Bishop, K., Krull, K.R., et al. (2015). Survivors of childhood cancer in the United States: prevalence and burden of morbidity. Cancer Epidemiology, Biomarkers and Prevention, 24(4):653-63.

[48] Porter, T.R., Shillcutt, S.K., Adams, M.S., Desjardins, G., Glas, K.E., Olson, J.J., et al. (2015). Guidelines for the use of echocardiography as a monitor for therapeutic intervention in adults: a report from the American Society of Echocardiography. Journal of American Society of Echocardiography, 28:40-56.

[49] Pradhan, B. and Kundu, D. (2014). Analysis of Interval-Censored Data with Weibull Lifetime Distribution. Sankhya B, 76,120-139.

[50] Rai, S. N. (2008). Analysis of occult tumor studies. In Handbook of cancer models with applications, World Scientific Press, Hackensack, NJ. 
[51] Rai, S.N. and Matthews, D.E. (1993). Improving the EM algorithm. Biometrics, 49(2):587-591.

[52] Rai, S.N. and Matthews, D.E. (1997). Discrete scale models for survival-sacrifice experiments. Applied Statistics, 48(1):93-109.

[53] Rai, S.N., Matthews, D.E., and Krewski, D.R. (2000). Mixed-scale models for survival/sacrifice experiments. The Canadian Journal of Statistics, 28(1):65-80.

[54] Rai, S.N., Pan, J., Sun, J., Hudson, M.M., and Srivastava, D.K. (2013). Estimating incidence rate on current status data with application to a Phase IV cancer trial. Communications in Statistics: Theory and Methods, 42:2417-2433.

[55] Robison, L.L. and Hudson, M.M. (2014). Survivors of childhood and adolescent cancer: life-long risks and responsibilities. Nature Reviews Cancer,14(1):61-70.

[56] Rubin, D.B., Stern, H., and Vehovar, V. (1995). Handing "don't know" survey responses: The case of the Slovenian plebiscite. Journal of the American Statistical Association, $90: 822-828$.

[57] Rubin, D.B. (1996). Multiple imputation after 18+ years (with discussion). Journal of the American Statistical Association, 91:473-489.

[58] Rubin, D.B. (1987). Multiple Imputation for Nonresponse in Surveys, John Wiley and Sons, Inc, New York.

[59] Ruiz-Castro, J.E. and Zenga, M. (2019). A general piecewise multi-state survival model: application to breast cancer. Statistical Methods and Applications, 29:813843.

[60] SAS/STATR14.1 User's Guide: The MI Procedure, SAS Institute Inc., Cary, NC, USA, 2015. 
[61] Satagopan, J., Ben-Porat, L., Berwick, M., Robson, M., Kutler, D., and Auerbach, A.D. (2004). A note on competing risks in survival data analysis. British Journal of Cancer, 91,1229-1235.

[62] Schafer, J.L. (1997). Analysis of Incomplete Multivariate Data, Chapman Hall, London.

[63] Sorensen, K., Levitt, G.A., Bull, C., Dorup, I., and Sullivan, I.D. (2003). Late anthracycline cardiotoxicity after childhood cancer: A perspective longitudinal study. Cancer, 97:1991-1998.

[64] Srivastava, D.K., Zhu, L., Hudson, M.M., Pan, J., and Rai, S.N. (2018). Robust estimation and inference on current status data with applications to Phase IV cancer trial. Journal of Modern Applied Statistical Methods, 17(1):18.

[65] Sun, J. (2006). The Statistical Analysis of Interval-Censored Failure Time Data, Springer, New York.

[66] Troxel, A., Guoguang, M., and Heitjan, D.F. (2004). An index of local sensitivity to nonignorability. Statistica Sinica, 14:1221-1237.

[67] Turnbull, B.W. (1976). The empirical distribution function with arbitrarily grouped, censored and truncated data. Journal of the Royal Statistical Society: Series B, 38:290-295.

[68] van Buuren, S. (2018). Flexible Imputation of Missing Data. Chapman and Hall/CRC, Florida, 2nd edition.

[69] van Buuren, S., Boshuizen, H.C., and Knook, D.L. (1999). Multiple imputation of missing blood pressure covariates in survival analysis. Statistics in Medicine, 18:681-694. 
[70] van Eeden, C. (1956). Maximum likelihood estimation of ordered probabilities. Indagationes Mathematicae, 18:444-455.

[71] van Eeden, C. (1957). Maximum likelihood estimation of partially ordered or completely ordered parameters. I, Nederal. Akad. Wetensch. Proc. Ser. A60, 128136; II, 201-211.

[72] van den Hout, A. (2020). Multi-state survival models for interval-censored data, Chapman and Hall/CRC. 


\section{APPENDIX}

Appendix 1: Detailed likelihood approach in a three-state model.

We adapt the following information from Rai et al. (2013).

From Figure 1.1, we define four likelihood functions corresponding with different scenario.

Death with No Cardiac Abnormality:

$$
L_{1}(t)=\lambda_{2}(t) Q(t)=\lambda_{2} e^{-\left(\lambda_{1}+\lambda_{2}\right) t}
$$

Alive with No Cardiac Abnormality:

$$
L_{2}(t)=Q(t)=e^{-\left(\lambda_{1}+\lambda_{2}\right) t}
$$

Death/Cardiac Failure with Cardiac Abnormality:

$$
L_{3}(t)=\int_{0}^{t} \lambda_{1} e^{-\left(\lambda_{1}+\lambda_{2}\right) u} \lambda_{3} e^{-\lambda_{3}(t-u)} d u=\frac{\lambda_{1} \lambda_{3}}{\lambda_{1}+\lambda_{2}-\lambda_{3}}\left(e^{-\lambda_{3} t}-e^{-\left(\lambda_{1}+\lambda_{2}\right) t}\right)
$$

Alive with Cardiac Abnormality:

$$
L_{4}(t)=\int_{0}^{t} \lambda_{1} e^{-\left(\lambda_{1}+\lambda_{2}\right) u} e^{-\lambda_{3}(t-u)} d u=\frac{\lambda_{1}}{\lambda_{1}+\lambda_{2}-\lambda_{3}}\left(e^{-\lambda_{3} t}-e^{-\left(\lambda_{1}+\lambda_{2}\right) t}\right)
$$


The complete log-likelihood function can be calculated as

$$
\begin{aligned}
l\left(\lambda_{1}, \lambda_{2}, \lambda_{3}\right)= & \sum_{i=1}^{n}\left[a_{i} \log L_{1}\left(t_{i}\right)+b_{i} \log L_{2}\left(t_{i}\right)+c_{i} \log L_{3}\left(t_{i}\right)+d_{i} \log L_{4}\left(t_{i}\right)\right] \\
= & \sum_{i=1}^{n} a_{i}\left[\log \lambda_{2}-\left(\lambda_{1}+\lambda_{2}\right) t_{i}\right]-\sum_{i=1}^{n} b_{i}\left(\lambda_{1}+\lambda_{2}\right) t_{i} \\
& +\sum_{i=1}^{n} c_{i}\left[\log \lambda_{1}+\log \lambda_{3}-\log \left(\lambda_{1}+\lambda_{2}-\lambda_{3}\right)+\log \left(e^{-\lambda_{3} t_{i}}-e^{-\left(\lambda_{1}+\lambda_{2}\right) t_{i}}\right)\right] \\
& +\sum_{i=1}^{n} d_{i}\left[\log \lambda_{1}-\log \left(\lambda_{1}+\lambda_{2}-\lambda_{3}\right)+\log \left(e^{-\lambda_{3} t_{i}}-e^{-\left(\lambda_{1}+\lambda_{2}\right) t_{i}}\right)\right]
\end{aligned}
$$

where $a_{i}=\delta_{i}\left(1-\gamma_{i}\right), b_{i}=\left(1-\delta_{i}\right)\left(1-\gamma_{i}\right), c_{i}=\delta_{i} \gamma_{i}, d_{i}=\left(1-\delta_{i}\right) \gamma_{i}$ are the indicators corresponding to observation type 1 to type 4 .

We define

$$
\delta_{i}= \begin{cases}1 & \text { if subject } i \text { dead or cardiac failure at time } t_{i} \\ 0 & \text { if subject } i \text { alive no cardiac failure at time } t_{i}\end{cases}
$$

and

$$
\gamma_{i}= \begin{cases}1 & \text { if subject } i \text { with abnormal value at time } t_{i} \\ 0 & \text { if subject } i \text { with normal value at time } t_{i}\end{cases}
$$

To help further simplify the equation, we define:

$$
\begin{gathered}
D_{1}=\sum_{i=1}^{n} a_{i}, D_{2}=\sum_{i=1}^{n} b_{i}, D_{3}=\sum_{i=1}^{n} c_{i}, D_{4}=\sum_{i=1}^{n} d_{i} \\
T_{1}=\sum_{i=1}^{n}\left(a_{i}+b_{i}+c_{i}+d_{i}\right) t_{i}, T_{2}=\sum_{i=1}^{n}\left(a_{i}+b_{i}\right) t_{i}
\end{gathered}
$$


Appendix 2: Derivation of components in the score function and information matrix.

$$
\begin{aligned}
& \frac{\partial l}{\partial \lambda_{1}}=-T_{2}+\left(\frac{1}{\lambda_{1}}-\frac{1}{\lambda_{1}+\lambda_{2}-\lambda_{3}}\right)\left(D_{3}+D_{4}\right)+\sum_{i=1}^{n} \frac{\left(c_{i}+d_{i}\right) t_{i}}{e^{\left(\lambda_{1}+\lambda_{2}-\lambda_{3}\right) t_{i}}-1} \\
& \left.\frac{\partial l}{\partial \lambda_{2}}=\frac{D_{1}}{\lambda_{2}}-T_{2}-\frac{D_{3}+D_{4}}{\lambda_{1}+\lambda_{2}-\lambda_{3}}\right)+\sum_{i=1}^{n} \frac{\left(c_{i}+d_{i}\right) t_{i}}{e^{\left(\lambda_{1}+\lambda_{2}-\lambda_{3}\right) t_{i}}-1} \\
& \frac{\partial l}{\partial \lambda_{3}}=\left(\frac{1}{\lambda_{3}}+\frac{1}{\lambda_{1}+\lambda_{2}-\lambda_{3}}\right) D_{3}+\frac{D_{4}}{\lambda_{1}+\lambda_{2}-\lambda_{3}}-\sum_{i=1}^{n} \frac{\left(c_{i}+d_{i}\right) t_{i}}{1-e^{\left.\lambda_{1}+\lambda_{2}-\lambda_{3}\right) t_{i}}} \\
& =\frac{D_{3}}{\lambda_{3}}+\frac{D_{3}+D_{4}}{\lambda_{1}+\lambda_{2}-\lambda_{3}}-T_{1}+T_{2}-\sum_{i=1}^{n} \frac{\left(c_{i}+d_{i}\right) t_{i}}{e^{\left(\lambda_{1}+\lambda_{2}-\lambda_{3}\right) t_{i}}-1} \\
& \frac{\partial^{2} l}{\partial \lambda_{1}^{2}}=\left(\frac{1}{\left(\lambda_{1}+\lambda_{2}-\lambda_{3}\right)^{2}}-\frac{1}{\lambda_{1}^{2}}\right)\left(D_{3}+D_{4}\right)-\sum_{i=1}^{n} \frac{\left(c_{i}+d_{i}\right) t_{i}^{2} e^{\left(\lambda_{1}+\lambda_{2}-\lambda_{3}\right) t_{i}}}{\left(e^{\left(\lambda_{1}+\lambda_{2}-\lambda_{3}\right) t_{i}}-1\right)^{2}} \\
& \frac{\partial l}{\partial \lambda_{1} \partial \lambda_{2}}=\frac{D_{3}+D_{4}}{\left(\lambda_{1}+\lambda_{2}-\lambda_{3}\right)^{2}}-\sum_{i=1}^{n} \frac{\left(c_{i}+d_{i}\right) t_{i}^{2} e^{\left(\lambda_{1}+\lambda_{2}-\lambda_{3}\right) t_{i}}}{\left(e^{\left(\lambda_{1}+\lambda_{2}-\lambda_{3}\right) t_{i}}-1\right)^{2}} \\
& \frac{\partial l}{\partial \lambda_{1} \partial \lambda_{3}}=-\frac{D_{3}+D_{4}}{\left(\lambda_{1}+\lambda_{2}-\lambda_{3}\right)^{2}}+\sum_{i=1}^{n} \frac{\left(c_{i}+d_{i}\right) t_{i}^{2} e^{\left(\lambda_{1}+\lambda_{2}-\lambda_{3}\right) t_{i}}}{\left(e^{\left(\lambda_{1}+\lambda_{2}-\lambda_{3}\right) t_{i}}-1\right)^{2}} \\
& \frac{\partial^{2} l}{\partial \lambda_{2}^{2}}=\frac{-D_{1}}{\lambda_{2}^{2}}+\frac{D_{3}+D_{4}}{\left(\lambda_{1}+\lambda_{2}-\lambda_{3}\right)^{2}}-\sum_{i=1}^{n} \frac{\left(c_{i}+d_{i}\right) t_{i}^{2} e^{\left(\lambda_{1}+\lambda_{2}-\lambda_{3}\right) t_{i}}}{\left(e^{\left(\lambda_{1}+\lambda_{2}-\lambda_{3}\right) t_{i}}-1\right)^{2}} \\
& \frac{\partial l}{\partial \lambda_{2} \partial \lambda_{3}}=-\frac{D_{3}+D_{4}}{\left(\lambda_{1}+\lambda_{2}-\lambda_{3}\right)^{2}}+\sum_{i=1}^{n} \frac{\left(c_{i}+d_{i}\right) t_{i}^{2} e^{\left(\lambda_{1}+\lambda_{2}-\lambda_{3}\right) t_{i}}}{\left(e^{\left(\lambda_{1}+\lambda_{2}-\lambda_{3}\right) t_{i}}-1\right)^{2}} \\
& \frac{\partial^{2} l}{\partial \lambda_{3}^{2}}=\frac{-D_{3}}{\lambda_{3}^{2}}+\frac{D_{3}+D_{4}}{\left(\lambda_{1}+\lambda_{2}-\lambda_{3}\right)^{2}}-\sum_{i=1}^{n} \frac{\left(c_{i}+d_{i}\right) t_{i}^{2} e^{\left(\lambda_{1}+\lambda_{2}-\lambda_{3}\right) t_{i}}}{\left(e^{\left(\lambda_{1}+\lambda_{2}-\lambda_{3}\right) t_{i}}-1\right)^{2}}
\end{aligned}
$$




\section{CURRICULUM VITA}

NAME:

ADDRESS:

EDUCATION:

\section{PUBLICATIONS:}

Rai, S., Qian, C.*, Pan, J., Seth, A., Srivastava, DK., \& Bhatnagar, A. (2020). Statistical design of Phase II/III clinical trials for testing therapeutic interventions in COVID-19 patients. BMC Medical Research Methodology, $20: 220$.

Qian, C., Rai, J., Pan, J., Wu, X., Bhatnagar, A., McClain, C., \& Rai, S. (2020). Target classification using machine learning approaches with applications to clinical studies. Biometrics $\mathscr{E}$ Biostatistics International Journal, 9(3):91-95. 
Rai, S., Qian, C.* , Pan, J., McClain, M., Eichenberger, M. R., McClain, C., \& Galandiuk, S. (2020). Statistical issues and group classification in plasma microRNA studies with data application. Evolutionary Bioinformatics.

Rai, S., Qian, C.* Pan, J., Rai, J., McClain, C., Bagaitkar, J., \& Egilmez, N. (2020). Microbiome data analysis with an application to a pre-clinical study using QIIME2: statistical considerations. Genes \& Diseases.

Liu, C., Qian, C., Qian, R., Liu, Z., Qiao, H., \& Zhang, Y. (2019). Numerical prediction of effective diffusivity in hardened cement paste between aggregates using different shapes of cement powder. Construction and Building Materials, 223:806816.

Shumway, B., Bernstein, M., Qian, C., Kulkarni, M., \& Rai, S. (2018). Effect of lecture attendance and prerequisite academic outcomes on oral pathology performance. Journal of Dental Education, 82(3):306312.

Qian, C., Caudill, S.B., \& Mixon Jr., F.G.(2016). Engaged in Teaching, and scholarship too: economics faculty productivity at national liberal arts colleges. International Journal of Pluralism and Economics Education, 7(4):360-372.

Qian, C., McClain, C., \& Rai, S. Improved confidence intervals 
for fixed term survival probabilities in a small two-arm trial. (Submitted)

Srivastava, DK., Qian, C.*, Pan, J., Hudson, M., Rai, S. Imputing partial status and estimating incidence rate in an illness-death model with application to a Phase IV cancer trial. (Submitted)

Burd, M., Gilbert, M., Rai, S., Qian, C., Rai, J., Knable, M., Sokolowski, D., Tse, W., Emmons, R., Hegazi, M., Figg, L. Efficacy and safety of Bortezomib for the treatment of steroid -refractory chronic graft versus host disease: a retrospective cohort analysis. (Submitted)

Dukka, H., Byrd, P., Qian, C., Baughman, G., Butt, S., \& Rai, S. Occupational percutaneous injuries and exposures in a dental teaching environment: a ten-year report. (Submitted)

* Equal first author.

PRESENTATIONS: Midwest Biopharmaceutical Statistics Workshop, Carmel, IN, May 2019.

Improved Confidence Intervals for Fixed Term Survival Probabilities in a Small Two-Arm Trial.

HONORS/AWARDS: University Fellowship, University of Louisville, 2017-2019 